\title{
Non-constant discounting, social welfare and endogenous growth with pollution externalities*
}

\author{
Francisco Cabo $^{a}$, Guiomar Martín-Herrán ${ }^{a} \dagger$ \\ María Pilar Martínez-García ${ }^{b}$ \\ ${ }^{a}$ IMUVa, Universidad de Valladolid, Spain ${ }^{\ddagger}$ \\ ${ }^{b}$ Universidad de Murcia, Spain ${ }^{\S}$
}

June 8, 2020

\begin{abstract}
We analyze the effect of non-constant discounting on economic growth and social welfare in an endogenous growth model with pollution externalities. For time-consistent agents, the existence of a balanced growth equilibrium is characterized and compared with the equilibrium under standard exponential discounting. A decaying instantaneous discount rate leads to slower growth in a centralized economy, while its effect for a competitive economy is ambiguous. Interestingly, when comparing the planned and the competitive equilibria, the assumption of non-constant discounting may imply greater social welfare under the market equilibrium. This counterintuitive result requires two conditions. First, pollution
\end{abstract}

${ }^{*}$ The authors have been partially supported by MEC under project ECO2014-52343-P, co-financed by FEDER funds.

${ }^{\dagger}$ Corresponding author: Guiomar Martín-Herrán, Dept. Economía Aplicada (Matemáticas), Facultad de Ciencias Económicas y Empresariales, Universidad de Valladolid, Avda. Valle Esgueva, 6, 47011 Valladolid, Spain. Tel: +34 983 423330. E-mail: guiomar@eco.uva.es

${ }^{\ddagger}$ Dept. Economía Aplicada (Matemáticas). Facultad de Ciencias Económicas y Empresariales, Universidad de Valladolid, Avda. Valle Esgueva, 6, 47011 Valladolid, Spain. E-mails: pcabo@eco.uva.es (F. Cabo), guiomar@eco.uva.es (G. Martín-Herrán)

${ }^{\S}$ Dept. Métodos Cuantitativos para la Economía y la Empresa, Facultad de Economía y Empresa, Universidad de Murcia, Campus del Espinardo, 30100 Murcia, Spain. E-mail: pilarmg@um.es 
externalities should lead the central planner to slow down growth to below the growth rate in the market economy. Secondly, individuals' degree of impatience should decrease sharply with the time distance from the present. Contrarily, when the centralized economy welfare dominates the market economy, introducing policy instruments is less effective than under constant discounting.

JEL Classification: D91, O44, C61.

Keywords: Non-constant discounting, endogenous growth, Social welfare, sustainability, environmental policies, time-consistent solutions.

\section{Introduction}

The optimal decisions of economic agents when confronted with intertemporal problems depend fundamentally on the way these agents discount the future. Ever since Samuelson (1937), the standard assumption in intertemporal decision making in economics is to discount at a constant rate, the so-called exponential discounting. ${ }^{1}$ A recent branch of the literature moves away from this assumption, considering individuals with a decreasing impatience more appropriate. This idea is highlighted in Laibson (1997) who pointed out that individuals are highly impatient about consumption in the near future but much more patient when confronted with decisions in the distant future.

As already pointed out by Ramsey (1928) and later by Strotz (1956), Pollak (1968) and Goldman (1980), with non-constant discounting, preferences change with time and therefore decisions taken at the present time will not necessarily be optimal if recomputed later on. This represents a problem of time inconsistency. If "naïve" individuals do not recognize this inconsistency they will continuously recompute their dynamic optimization problems, and their optimal decisions are time inconsistent. In contrast, if they recognize the inconsistency two situations are possible. In certain occasions the present generation has the power to commit the behavior of future generations and is certain that its optimal decisions will be maintained over time. If no such commitment device exists individuals behave sophisticatedly. The present generation must choose its optimal course of action over time taking into account the possibility of future disobedience. In other words, it must add the behavior of future generations as a constraint in its decision making problem. The representative individual in the present generation regards his infinitely

\footnotetext{
${ }^{1}$ Later, Strotz (1956) proved that exponential discounting is the only discount function which guarantees time consistency.
} 
many future selves as separate individuals against whom he is playing a game. The Nash equilibria of this intergenerational game give rise to time-consistent optimal decisions.

With non-constant time preferences, when commitment is not feasible, the solution to the game played by a sophisticated central planner against his future selves needs not be Pareto-improving when compared with the Nash equilibrium of the game played by individuals in a decentralized or market solution. This result has been highlighted in the literature of Neoclassical growth by Krusell et al. (2002), based on the idea that the planner acknowledges his effect on the returns to savings, while these returns are considered constant by price-taker consumers. Thus, the rate of return is decreasing for the planner and constant for competitive agents. In consequence, the latter saves more and the competitive economy grows faster (towards the steady state). Therefore, the decentralized economy ends up providing greater social welfare. ${ }^{2}$ While this result is obtained considering quasi-geometric discounting, Hiraguchi (2014) proves that it remains valid for a general non-constant discounting function. ${ }^{3}$

Our main finding shows that a faster growth and a Pareto improving market solution can also arise in an endogenous growth model with environmental externalities. To that aim, following the seminal paper by Smulders and Gradus (1996), the environmental quality is associated with two externalities: it increases utility as well as the productivity of the direct factors of production. Agents are consumption-driven individuals and also give entrance to the amenities stemming from the environmental quality. Pollution is a by-product of production which negatively affects productivity and which can be reduced by abatement. In this endogenous growth model, a balanced path equilibrium is feasible if the optimal consumption and abatement per unit of capital remain constant. In the framework of an intergenerational game, this corresponds to a current individual who correctly anticipates the future behavior of all his future selves, restricted to the assumption of consumption and abatement strategies linear in the capital stock. That is, constant expected propensities to consume and to abate out of capital (which, in this model, defines wealth). Likewise, future generations also play this same game against their future selves, which generates an infinite sequence of identical optimal consumption and abatement strategies. Under stationarity, optimal and anticipated decisions coincide and hence expectations are fulfilled giving rise to an equilibrium, which will be accepted

\footnotetext{
${ }^{2}$ Along the same line, this result of group inefficiency can be also obtained in the case of asymmetric players (with different discount rates), as shown in Marín-Solano (2015) and Castañer et al. (2016).

${ }^{3}$ However, more recently, Hiraguchi (2016) proves that the competitive economy never outperforms the centralized economy in a two-sector endogenous growth.
} 
by all future cohorts. Thus, considering agents who discount the future at non-constant rates, we analyze Markov-perfect Nash equilibria in linear and stationary strategies, called a stationary perfect equilibrium.

To compare the social welfare under the central planner and the market economy, we define a central planner as a representative consumer who shares the same time preferences with his cohort and internalizes the two externalities caused by pollution. If the externality from the producers' denial of the effect of their own generated pollution on production is relatively small in comparison with the negative externality of pollution on utility, then the central planner would welcome a deceleration in economic growth to slow down pollution. This is not taken into consideration in the market economy which, in consequence, might grow faster than the centralized economy. Whether a faster growth implies a higher social welfare in the market economy crucially depends on how the agents discount the future.

When considering individuals whose impatience decreases with the time distance from the present, a fast growing market economy provides higher welfare than a planned economy if the individuals' level of impatience decreases sharply. The intuition is that assuming an identical overall level of impatience, the faster the decay in the instantaneous discount rate, the greater the weight given to the distant future with respect to the near future. Thus, for strongly long-run oriented individuals, if the market economy grows faster, social welfare is also higher. A higher valuation of the distant future makes more rewarding economic growth and hence more attractive the market economy in social welfare terms. The result is proved for log-utility, but we cannot prove it for a more general isoelastic utility. In that case, a similar result is obtained in a parallel research for a simpler model with no abatement and where pollution only affects utility but not production (see Cabo et al. 2019).

Conversely, when the central planner's solution welfare dominates the decentralized market economy it is appropriate to levy taxes on production and pollution, or to grant a subsidy on abatement activities to bring the market toward the central planner's equilibrium. We compare the effectiveness of these policies under constant and nonconstant discounting. When individuals discount at a non-constant rate the policies are equally effective under log-utility, but less effective for an isoelastic utility with an elasticity of intertemporal substitution lower one. Thus, lower efficiency is proven starting from an initial situation of a pure free market economy.

Additionally, we study how the assumption of non-constant discounting modifies 
the balanced path equilibrium under exponential discounting analyzed in Smulders and Gradus (1996), both in the centralized and the market economies. For a log-utility, a current marginal increment in the capital stock will have a constant effect on all future utilities along a balanced growth path. Under the identical overall impatience assumption the value of all these ongoing effects is the same under constant and nonconstant discounting, and so the economy grows at the same rate under both specifications. However, in the case of an isoelastic utility with an elasticity of intertemporal substitution below one, a current marginal increment in capital has decreasing ongoing effects. Because it is in the short run where these marginal effects are stronger and non-constant-discounting individuals value less the short run, then these agents undervalue the accumulated effect of current changes in capital on all ongoing utilities, with respect to constant-discounting individuals. For this reason, a decreasing rate of time preference implies slower growth and higher propensity to consume. Moreover, in the central planner's solution it also leads to higher investments in abatement.

The paper is organized as follows. Section 2 presents a short review of the literature. Section 3 summarizes the main ingredients of the well-established model of pollution abatement and endogenous growth by Smulders and Gradus (1996) and characterizes the Markov-perfect Nash equilibrium of the intergenerational game played by the central planner under non-constant discounting. The results are contrasted with the standard assumption of constant discounting. The decentralized economy and the appropriate environmental policies are presented in Section 4. Section 5 analyzes the conditions under which the market economy welfare dominates the central planner's solution, and provides an example for a specific family of discount functions. In contrast, when the centralized solution welfare dominates the market economy, the effectiveness of taxes/subsidy is also compared for constant and non-constant discounting. Finally, Section 6 concludes. Most proofs are presented in the Appendix.

\section{Literature revision}

An important strand of the literature supports the appropriateness of time-varying discount rates based on theoretical and empirical grounds (see, Frederick et al. (2002) and DellaVigna (2009) for a survey). However, this opinion is not unanimous, with some criticisms mostly supported by empirical evidence (see, for example, Andersen et al. (2014)). Besides, there exists an additional debate on whether individuals do or do 
not recognize the inconsistency of current decisions when recomputed by future selves. There is no clear consensus in the literature on which type of agent more truthfully resembles real individuals. Some authors (like Caliendo and Aadland (2007), Findley and Caliendo (2014) or Farzin and Wedner (2014)) support the hypothesis that individuals are indeed unaware of their future impatience. In contrast, we align ourselves with others (like Barro (1999), Karp (2007), or Karp and Tsur (2011)) assuming sophisticated consumers, whose optimal consumption paths are time consistent.

The literature on economic growth and non-constant discounting was initiated by Barro (1999). For a Neoclassical growth model he shows that non-constant (quasihyperbolic) discounting is observationally equivalent to exponential discounting when a log-utility function and sophisticated agents are considered. This equivalence is not necessarily true, as proved first in Farzin and Wedner (2014) when the elasticity of intertemporal substitution differs from one (assuming naïve consumers), and secondly, in Cabo et. al (2016), when a non-constant discount function other than the quasihyperbolic is considered (assuming sophisticated consumers). Similarly, for Ak-type endogenous growth models, Strulik (2015) proves that hyperbolic and exponential discounting are observationally equivalent for log-utility and naïve consumers. Moreover he proves strong equivalence, which was defined as having the same growth rate under the assumption of an identical overall impatience. However, as proved in Cabo et. al (2015), isoelastic, although not logarithmic, utility preserves observational equivalence but not strong equivalence, regardless of whether individuals are naive or sophisticated. This literature, which highlights the importance of the elasticity of intertemporal substitution, does not focus on welfare comparisons. Indeed, observational equivalence does not preclude differences in welfare, as already commented by Krusell et al. (2002) or Hiraguchi (2014) for a Neoclassical growth model.

The literature on environmental economics and natural resource management has already shown an interest in the rationale for the use of non-constant discounting. Groom et al. (2005) revise the different reasons that justify the use of declining discount rates, and the main criticisms of this assumption for environmental problems, in particular for global warming. The pros and cons of hyperbolic discounting are also reviewed in Hepburn et al. (2010), which highlights the time inconsistency problem for a model of natural resource management. A seminal work studying non-constant discounting in an endogenous growth model with a renewable natural resource is presented in $\mathrm{Li}$ and Lofgren (2000), which analyzes the sustainability of economic growth based on the 
Chichilnisky criterion (Chichilnisky 1996). For the particular case of global warming, the effect of hyperbolic discounting on the optimal policies has been analyzed from various perspectives. The time consistent equilibria are characterized for a discrete time formulation in Karp (2005) and Fuji and Karp (2008), and for continuous time in Karp and Tsur (2011). More recently, Gerlagh and Liski (2017) revisit the implications of non-constant discounting for climate policies. Our analysis is a contribution to the literature on environmental economics and non-constant discounting by considering an endogenous growth model in which the quality of the environment affects productivity and consumers' utility.

\section{A central planner with non-constant discounting}

This paper revisits the endogenous growth model with pollution abatement presented in Smulders and Gradus (1996). The economy produces a final output, $Y$, using capital, $K$, understood as a broad measure of physical and human capital, knowledge and technology. Productivity of capital is negatively affected by net pollution, $P$, which is the result of two opposing forces. On the one hand, pollution is a by-product of the economic activity, and hence it shows a positive relation with the capital stock. On the other hand, the economy can carry out abatement activities, $A$, to reduce pollution. Smulders and Gradus (1996) consider the following Cobb-Douglas production and pollution functions.

$$
Y(P, K)=B P^{-\alpha} K^{\beta}, \quad P(A, K)=A^{-\gamma} K^{\lambda}, \quad \alpha, \beta, \gamma, \lambda>0
$$

Households utility, $U(C, P)$, is an isoelastic utility function which depends positively on consumption and negatively on pollution, that is,

$$
U(C, P)= \begin{cases}\frac{\left(C P^{-\phi}\right)^{1-1 / \sigma}-1}{1-1 / \sigma} & \text { if } \sigma \neq 1 \\ \ln C-\phi \ln P & \text { if } \sigma=1 .\end{cases}
$$

Parameter $\sigma \in(0,1]$ is the constant elasticity of intertemporal substitution and $\phi>$ 0 can be interpreted as a measure of the environmental concern. The elasticity of intratemporal substitution between consumption and pollution equals one. Assuming constant population normalized to one, all variables are in per capita terms.

A balanced growth path is characterized by a constant output per unit of capital 
and constant propensities to consume and to abate out of wealth. ${ }^{4}$ Hence, the ratios $\psi_{\mathrm{C}}=C / K$ and $\psi_{\mathrm{A}}=A / K$ remain constant. From (1), writing the output as a function of capital, it follows that a balanced growth path is feasible if the following conditions are satisfied:

$$
\begin{aligned}
& \beta+\alpha(\gamma-\lambda)=1 \\
& \lambda \leq \gamma
\end{aligned}
$$

Then, the output per unit of capital, $y=Y / K$, can be defined as a function of the propensity to abate:

$$
y\left(\psi_{\mathrm{A}}\right)=B \psi_{\mathrm{A}}^{\alpha \gamma}
$$

\subsection{The central planner's solution}

Smulders and Gradus (1996) study this model under exponential discounting. In contrast, in the present paper, this stylized model is analyzed assuming that individuals discount the future at a decreasing rate: their degree of impatience decreases with the time distance from the present. As Krusell et al. (2002) acknowledge, when successive cohorts disagree it is difficult to determine a suitable notion for central planner. However, we assume (as in Krusell et al. (2002) and Hiraguchi (2014)) that the central planner's preferences are the same as those of the current self.

Following the literature on sophisticated equilibria (Phelps and Pollak 1968, Leininger 1986 and Sorger 2004) we consider that at any time $t$, the current central planner, or $t$-agent, plays a game with his future selves. At this time he chooses the instantaneous consumption and abatement taking into account that these decisions will affect all his future selves and assuming that all these future selves play the same linear strategies $C_{t}(\tau)=\psi_{\mathrm{C}} K_{t}(\tau)$ and $A_{t}(\tau)=\psi_{\mathrm{A}} K_{t}(\tau), \tau \geq t$, with $\psi_{\mathrm{C}}$ and $\psi_{\mathrm{A}}$ constant. Thus, at each time $t$ the central planner maximizes the lifetime utility of the representative consumer (t-agent), subject to the dynamic evolution of the capital stock from this instant on. By stationarity, in equilibrium all future selves use the same strategy, which coincides with the optimal strategy of the $t$-agent, rendering the solution time consistent. Under condition (3) and linear strategies, this equilibrium is a balanced growth path.

\footnotetext{
${ }^{4}$ Because labor is ignored, individuals' wealth is exclusively defined by their assets, which in aggregate terms are equal to the capital stock.
} 
The optimization problem faced by this sophisticated planner reads:

$$
\begin{array}{ll} 
& \max _{C_{t}, A_{t}} \int_{t}^{\infty} U\left(C_{t}(\tau), P_{t}(\tau)\right) \theta(\tau-t) d \tau, \\
\text { s.t.: } & \dot{K}_{t}(\tau)=B P_{t}^{-\alpha}(\tau) K_{t}^{\beta}(\tau)-C_{t}(\tau)-A_{t}(\tau), \quad K_{t}(t)=K_{t}, \\
& C_{t}(\tau)=\psi_{\mathrm{C}} K_{t}(\tau), \quad A_{t}(\tau)=\psi_{\mathrm{A}} K_{t}(\tau), \quad \tau \geq t .
\end{array}
$$

where the utility function is given by $(2)$ and $P_{t}(\tau)=A_{t}^{-\gamma}(\tau) K_{t}^{\lambda}(\tau)$. In this problem $t$ represents current date, and $j=\tau-t$ the time distance from the present. Note that by (8), choosing $C_{t}$ and $A_{t}$ is equivalent to determine $\psi_{\mathrm{C}}$ and $\psi_{\mathrm{A}}$.

Time preference is measured by the discount function $\theta(j) \geq 0$, which is not a function of current time, but of the time distance from the present, $j$. The discount function satisfies $\theta(j)>0, \dot{\theta}(j)<0, \forall j \geq 0$ and $\theta(0)=1$. The instantaneous discount rate $\rho(j) \doteqdot-\dot{\theta}(j) / \theta(j)$ is not a constant, but decreases with the time distance from the present: $\rho(j)>0, \dot{\rho}(j)<0, \forall j \geq 0$. Finally, $\lim _{t \rightarrow+\infty} \rho(j)$ can be strictly positive (quasi-hyperbolic discounting) or null (hyperbolic discounting). The improper integral $\int_{0}^{\infty} \theta(j) d j$ can be interpreted as a measure of the overall impatience. Along the paper, we assume that this integral is convergent.

Following Karp (2007), the optimality of a stationary solution of problem (6)-(8) must satisfy the following Bellman equation, ${ }^{5}$

$$
\begin{aligned}
\int_{t}^{\infty} & U\left(C_{t}^{*}(\tau), P_{t}^{*}(\tau)\right) \theta(\tau-t) \rho(\tau-t) d \tau= \\
& \max _{C_{t}, A_{t}}\left\{U\left(C_{t}, P\left(A_{t}, K_{t}\right)\right)+W^{\prime}\left(K_{t}\right)\left[Y\left(P\left(A_{t}, K_{t}\right), K_{t}\right)-C_{t}-A_{t}\right]\right\},
\end{aligned}
$$

where $C_{t}^{*}(\tau)=\psi_{\mathrm{C}}^{*} K_{t}^{*}(\tau)$ is the consumption and $P_{t}^{*}(\tau)=P^{*}\left(\psi_{\mathrm{A}}^{*} K_{t}^{*}(\tau), K_{t}^{*}(\tau)\right)$ the pollution along the optimal path for the $t$-agent, and $W\left(K_{t}\right)=\int_{t}^{\infty} U\left(C_{t}^{*}(\tau), P_{t}^{*}(\tau)\right) \theta(\tau-$ $t) d \tau$, denotes the value function. Subscript $t$ relates to the $t$-agent, who solves the maximization problem starting at current time $t$. In the RHS of (9) the argument for these variables is this current time $t$. For the ease of presentation we omit this argument when no confusion can arise.

First-order conditions for optimality establish that

$$
\begin{aligned}
& U_{\mathrm{C}_{t}}^{\prime}=W^{\prime}\left(K_{t}\right), \\
& U_{\mathrm{P}_{t}}^{\prime} P_{\mathrm{A}_{t}}^{\prime}=W^{\prime}\left(K_{t}\right)\left(1-Y_{\mathrm{P}_{t}}^{\prime} P_{\mathrm{A}_{t}}^{\prime}\right) .
\end{aligned}
$$

\footnotetext{
${ }^{5} \mathrm{~A}$ method to find a time-consistent solution with non-constant discounting is proposed in Karp (2007) for an infinite time horizon and in Marín-Solano and Navas (2009) for a finite time problem.
} 
And hence: $U_{\mathrm{P}_{t}}^{\prime} P_{\mathrm{A}_{t}}^{\prime}=U_{\mathrm{C}_{t}}^{\prime}\left(1-Y_{\mathrm{P}_{t}}^{\prime} P_{\mathrm{A}_{t}}^{\prime}\right)$, which can be rewritten as

$$
A_{t}=\frac{\psi_{\mathrm{A}} \phi \gamma}{\psi_{\mathrm{A}}-\alpha \gamma y\left(\psi_{\mathrm{A}}\right)} C_{t} \quad \Leftrightarrow \quad \psi_{\mathrm{C}}=\psi_{\mathrm{A}} \frac{1-\alpha \gamma B \psi_{\mathrm{A}}^{\alpha \gamma-1}}{\phi \gamma},
$$

establishing a positive relation between the propensity to consume and to abate, provided that $1>\alpha \gamma$, which is assumed henceforth. Note that abatement can not exceed output, which from (5) implies an upper bound for $\psi_{\mathrm{A}}$. Moreover, abatement and consumption must be simultaneously positive, which from (12) defines a lower bound for $\psi_{\mathrm{A}}$. From these two constraints it follows:

$$
\psi_{\mathrm{A}} \in\left((\alpha \gamma B)^{\frac{1}{1-\alpha \gamma}}, B^{\frac{1}{1-\alpha \gamma}}\right]
$$

Moreover, as the following proposition proves, the optimality of a balanced growth path requires satisfying a modified Ramsey rule. This rule is obtained under the assumption that the elasticity of intertemporal substitution, $\sigma$, is lower than or equal to one. This assumption of an income effect stronger than or equal to the substitution effect is often referred to as the empirically relevant case.

Proposition 1 For $\sigma \leq 1$, the growth rate of the central planner's economy along a balanced path equilibrium for problem (6)-(8) must satisfy the modified Ramsey rule:

$$
g \doteqdot \frac{\dot{C}}{C}=\frac{\beta y\left(\psi_{A}\right)-\psi_{A} \frac{\lambda}{\gamma}-\Delta(g, \eta)}{1+\eta}
$$

with

$$
\eta=\frac{1-\sigma}{\sigma}[1+\phi(\gamma-\lambda)] \geq 0
$$

and

$$
\Delta(g, \eta)=\int_{0}^{\infty} \rho(j) \omega(j) d j, \text { with } \omega(j)=\frac{\theta(j) e^{-\eta g j}}{\int_{0}^{\infty} \theta(i) e^{-\eta g i} d i} \in(0,1)
$$

Proof. See the Appendix

Note that for $\sigma=1$, then $\eta=0$ and $\Delta(g, 0)=\int_{0}^{\infty} \rho(j) \omega_{0}(j) d j=\hat{\rho}$, with $\omega_{0}(j)=$ $\theta(j) / \int_{0}^{\infty} \theta(i) d i \in(0,1)$. Then, (14) particularizes as: ${ }^{6}$

$$
g^{1}=\beta y\left(\psi_{\mathrm{A}}\right)-\psi_{\mathrm{A}} \frac{\lambda}{\gamma}-\hat{\rho}
$$

with $\hat{\rho}=\left[\int_{0}^{\infty} \theta(j) d j\right]^{-1}$.

\footnotetext{
${ }^{6}$ Here and henceforth, the particular case $\sigma=1$ is highlighted with a superscript.
} 
Remark 2 Note that if $g>0$, the expression of $\Delta(g, \eta)$ is well defined under the following alternative assumptions: a) for $\sigma<1(\eta>0)$; b) for $\sigma=1(\eta=0)$, and a convergent improper integral $\int_{0}^{\infty} j \theta(j) d j$; c) for $\sigma>1(\eta<0)$ and $\lim _{t \rightarrow+\infty} \rho(j)>-\eta g$. This latter condition translates into an upper bound for the elasticity of intertemporal substitution above one, as it also occurs in economic growth problems under constant discounting. However, the latter case is outside the scope of this paper.

\subsection{Comparing constant and non-constant discounting}

In what follows, and in order to compare non-constant discounting with the standard results with exponential discounting, we need to guarantee that the dissimilarities are not due to different degrees of impatience (see, for example, Strulik 2015 and Cabo et al. 2015). Therefore, both discounting methods have to be controlled to show identical overall impatience. That is:

$$
\int_{0}^{\infty} e^{-\hat{\rho} j} d j=\int_{0}^{\infty} \theta(j) d j, \quad \Longleftrightarrow \hat{\rho}=\left[\int_{0}^{\infty} \theta(j) d j\right]^{-1} .
$$

It is worth noting that under non-constant discounting the short run is less valued and the long run more valued than under exponential discounting (see Figure 1). This is due to the fact that the instantaneous discount rate is initially large and decreases with the time distance from the present, together with the assumption of identical overall impatience.

\section{Insert Figure 1 about here}

In the standard case of constant discounting, that is, $\rho(j) \equiv \hat{\rho}$, the integral $\Delta(g, \eta)$ boils down to $\hat{\rho}$. Therefore, the growth rate along the balanced path in expressions (14) or (17) reduces to the Ramsey rule found in Smulders and Gradus (1996): ${ }^{7}$

$$
g_{\hat{\rho}}=\frac{\beta y\left(\psi_{\mathrm{A}}\right)-\psi_{\mathrm{A}} \frac{\lambda}{\gamma}-\hat{\rho}}{1+\eta}, \quad g_{\hat{\rho}}^{1}=\beta y\left(\psi_{\mathrm{A}}\right)-\psi_{\mathrm{A}} \frac{\lambda}{\gamma}-\hat{\rho},
$$

where subscript $\hat{\rho}$ refers to the standard exponential discounting. The role played by the constant temporal discount rate in (19) is played by $\Delta(g, \eta)$ in the case of non-constant discounting, (14). Therefore, the latter is henceforth denoted as the effective rate of time preference.

\footnotetext{
${ }^{7}$ These authors present the Ramsey rule as a function of the abatement-spending ratio, while we present them as function of the propensity to abate. Nonetheless, both expressions are identical.
} 
While the Ramsey rule with constant discounting explicitly defines the growth rate as a function of the propensity to abate, the equivalent modified Ramsey rule in the case with non-constant discounting in (14) implicitly defines the growth rate of the economy. The implicit relationship is proven in Proposition 14 in the Appendix.

The social rate of return of the economy can be written as the same function of the propensity to abate, regardless of whether the future is discounted at a constant or at a decreasing rate:

$$
r\left(\psi_{\mathrm{A}}\right)=\beta y\left(\psi_{\mathrm{A}}\right)-\psi_{\mathrm{A}} \frac{\lambda}{\gamma} .
$$

The accumulated flow of discounted utility along a balanced path can be written as,

$$
\begin{aligned}
& \int_{t}^{\infty} U\left(C_{t}(\tau), P_{t}(\tau)\right) \theta(\tau-t) d \tau= \\
& \begin{cases}\frac{\sigma}{(1-\sigma) \hat{\rho}}-\sigma \frac{\left(\psi_{\mathrm{C}} \psi_{\mathrm{A}}^{\phi \gamma}\right)^{1-\frac{1}{\sigma}}}{1-\sigma} K_{t}^{-\eta} \Omega(g, \eta) & \text { if } \sigma<1, \\
\frac{(1+\phi(\gamma-\lambda))\left[\ln \left(K_{t}\right)+g \bar{J}_{0}\right]}{\hat{\rho}}+\frac{\ln \left(\psi_{\mathrm{C}} \psi_{\mathrm{A}}^{\phi \gamma}\right)}{\hat{\rho}} & \text { if } \sigma=1,\end{cases}
\end{aligned}
$$

where

$$
\Omega(g, \eta)=\int_{0}^{\infty} \theta(j) e^{-\eta g j} d j, \quad \bar{J}_{0}=\int_{0}^{\infty} j \theta(j) d j .
$$

For the case of constant discounting expression (21) remains valid with

$$
\Omega_{\hat{\rho}}(g, \eta)=\int_{0}^{\infty} e^{-(\eta g+\hat{\rho}) j} d j=\frac{1}{\eta g+\hat{\rho}} \leq \frac{1}{\hat{\rho}} .
$$

$\Omega(g, \eta)$ and $\Omega_{\hat{\rho}}(g, \eta)$ collect the total effect of a marginal increment in current capital on all future utility, as the aggregated sum of the discounted value of ongoing future marginal effects on utility. If the income and the substitution effects are identical (under $\log$-utility $\eta=0)$, or there is no growth $(g=0)$, current marginal effects remain constant at all future times. Therefore, the global effect would be exclusively determined by the overall level of impatience, $\Omega(g, 0)=\Omega(0, \eta)=\Omega_{\hat{\rho}}(g, 0)=\Omega_{\hat{\rho}}(0, \eta)=1 / \hat{\rho}$. However, if $\sigma<1$, the marginal effect of current increments in capital on future utility along a balanced path, $e^{-\eta g j}$, decreases with the time distance from the present. Moreover, due to the assumption of an identical overall level of impatience, individuals who discount the future at a non-constant discount rate give less value to the short run (where the marginal effect is stronger) and more value to the long run (where it is weaker) than standard individuals with constant discounting (see Figure 1). These two facts lead to the following result: 
Corollary 3 Assuming an elasticity of intertemporal substitution lower than one in a growing economy, a central planner who discounts the future at a non-constant rate underestimates the effect of current savings on ongoing utility. This underestimation disappears if the growth rate of the economy tends to zero or the elasticity of intertemporal substitution tends to one:

$$
\Omega(g, \eta)<\Omega_{\hat{\rho}}(g, \eta), \quad g, \eta>0, \quad \text { and } \quad \lim _{\sigma \rightarrow 1^{-}} \Omega(g, \eta)=\lim _{g \rightarrow 0^{+}} \Omega(g, \eta)=\frac{1}{\hat{\rho}} .
$$

It is now possible to give an interpretation of the effective rate of time preference, $\Delta(g, \eta)$, defined in (16) as a weighted mean of the decaying instantaneous discount rates, $\rho(j)$, with weights $\omega(j)$ satisfying $\int_{0}^{\infty} \omega(j) d j=1$. The weight $\omega(j)$ represents the value that a marginal rise in current capital will have on the utility $j$ instants from now on (in relative terms to the accumulated effect on ongoing utility at all future times). The next proposition compares the effective discount rate under constant and non-constant discounting. It distinguishes between the extreme cases $\sigma=1$ or $g=0$, and the general case with $\sigma<1$ and $g>0$.

\section{Proposition 4}

$$
\begin{aligned}
& \text { If } \sigma<1 \text { and } g>0 \text { then } \Delta(g, \eta)>\hat{\rho}, \\
& \text { If } \sigma=1 \text { or } g=0 \text { then } \Delta(g, 0)=\Delta(0, \eta)=\hat{\rho} .
\end{aligned}
$$

In the extreme cases of $\sigma=1$ or $g=0$, there is no decay in the marginal effect of current capital on future utility, ${ }^{8}$ and taking into account the assumption of identical overall impatience, the effective discount rate coincides with the constant discount rate $\hat{\rho}$. In consequence, in these two extreme cases the propensity to consume out of wealth coincides under constant and non-constant discounting.

In the general case of $\sigma<1$ and a positive growth rate, current effects of present decisions on future utility decrease with the time distance from the present. Therefore, the marginal effect of current savings on future consumption is under-valued with respect to the case of exponential discounting, implying a higher propensity to consume out of wealth. ${ }^{9}$ Consequently, as stated in the following proposition and Figure 2, the modified Ramsey rule under non-constant discounting lies below the Ramsey curve with constant

\footnotetext{
${ }^{8}$ The weights $\omega(j)$ only depend on the discount function $\theta(j) / \int_{0}^{\infty} \theta(j) d j$.

${ }^{9}$ From (61) and Lemma 13 it follows that the propensity to consume out of wealth increases with the growth rate of the economy, driven by a stronger income than substitution effect. And this increment is more intense the higher $\eta$ is, i.e. the lower below one $\sigma$ is.
} 
discounting. That is, a lower willingness to grow for any propensity to abate. This is equivalent to having an effective discount rate greater than $\hat{\rho}$ in (14), which is "as if" the central planner discounted the future at a higher but constant rate, $\Delta(g, \eta)>\hat{\rho}$. Moreover, the modified Ramsey rule displays an inverted U-shape as a function of the propensity to abate.

Proposition 5 When $\sigma<1$ and $g>0$, the modified Ramsey curve with non-constant discounting in (14) is an inverted U-shaped curve in $\psi_{A}$. This curve lies below and is less steep than the Ramsey curve under exponential discounting.

For $\sigma=1$, the Ramsey curves under constant and non-constant discounting coincide.

Proof. See the Appendix

These results are displayed in Figure 2. The standard Ramsey curve in the case of constant discounting is displayed by the inverted U-shaped dashed blue curve. The modified Ramsey rule under non-constant discounting, in solid red, lies below and although still inverse U-shaped, it shows gentler increments and decrements with $\psi_{\mathrm{A}}$.

To fully characterize the equilibrium growth rate, equation (7) should also be taken into account. Replacing (12) into (7) the growth rate of the capital stock can be written as

$$
g=y\left(\psi_{\mathrm{A}}\right)-\psi_{\mathrm{C}}-\psi_{\mathrm{A}}=\left(1+\frac{\alpha}{\phi}\right) y\left(\psi_{\mathrm{A}}\right)-\frac{1+\phi \gamma}{\phi \gamma} \psi_{\mathrm{A}}
$$

This curve (dashed-dotted black curve in Figure 2) represents the balanced budget or the feasible set of balanced paths in the terminology of Smulders and Gradus (1996). The equilibrium is displayed by the red dot, $\left(\psi_{\mathrm{A}}^{*}, g^{*}\right)$, in Figure 2. Likewise, the figure also plots the equilibrium under constant discounting, $\left(\psi_{\mathrm{A} \hat{\rho}}^{*}, g_{\hat{\rho}}^{*}\right)$, as the cross between the budget constraint in (27) and the Ramsey rule in (19). These equilibria can lie in the upward- or the downward-sloping part of the Ramsey curves. In either case, the assumption of non-constant discounting implies a balanced growth path characterized by a lower growth rate and a greater propensity to abate. This result is established in next proposition.

Proposition 6 For $\sigma<1$, under the assumption of identical overall impatience in (18), if the future is discounted at a decreasing rate, the central planner's optimal growth rate would be lower and the propensity to abate and consume higher than if constant discounting applies:

$$
g^{*}<g_{\hat{\rho}}^{*}, \quad \psi_{A}^{*}>\psi_{A \hat{\rho}}^{*}, \quad \psi_{C}^{*}>\psi_{C \hat{\rho}}^{*} .
$$

For $\sigma=1$ the two equilibria coincide, $g^{1 *}=g_{\hat{\rho}}^{1 *}, \psi_{A}^{1 *}=\psi_{A \hat{\rho}}^{1 *}$ and $\psi_{C}^{1 *}=\psi_{C \hat{\rho}}^{1 *}$. 
Insert Figure 2 about here

Proof. See the Appendix

Remark 7 Following the notation in Strulik (2015), we have proved that if $\sigma<1$ the models with constant and non-constant discounting are observationally equivalent in the sense that under both discounting methods along the balanced path the growth rate of the economy is constant, and abatement, consumption, capital and output grow at the same constant rate, while pollution decreases at a constant rate. However, the models are not strongly equivalent because they lead to different economic growth rates, despite the assumption of identical overall impatience.

\section{The market economy}

This section presents the market or decentralized economy considering that time preferences are identical to those previously explained for the central planner. Recall that the central planner has been interpreted as a representative agent who internalizes the two externalities caused by pollution. Consumption decisions are taken by dynamic consumers whose time preferences decrease with the time distance from the present. Abatement is chosen by static maximizing firms. Once the decentralized equilibrium is characterized, we compare the growth rate and the social welfare with those associated with the central planner's solution. When appropriate, we also analyze the effect of the environmental policies put into practice by a benevolent government and compare them against the case of exponential discounting.

We consider an economy populated by a large number of identical firms of measure one. Firm $i$ uses only capital as input. ${ }^{10}$ Production activities (measured by the capital involved) of firm $i$ generates pollution, $P_{i}$, which can be reduced by the abatement activities carried out by this firm $A_{i}$ :

$$
P_{i}=A_{i}^{-\gamma} K_{i}^{\lambda}
$$

\footnotetext{
${ }^{10}$ Alternatively, we could also include labor as an input. Endogenous growth could be attained assuming learning by doing and knowledge spillovers as, for example, explained in Barro and Sala-iMartin (2004). To have a better insight on the results of the model, we analyze a simpler version without labor, hence ignoring the knowledge externality and focusing only on the two externalities caused by pollution (on production and utility).
} 
Following Smulders and Gradus (1996), the aggregate pollution generated by all firms, $P=\int_{0}^{1} P_{i} d i$ negatively affects each firm's productivity. However, each particular firm only internalizes part of its own pollution. Therefore, firm's $i$ production is not exclusively a function of the capital input, but also of global pollution, and in particular its own pollution which is partially internalized:

$$
Y_{i}=B K_{i}^{\beta} P_{i}^{-\tilde{\alpha}} P^{-(\alpha-\tilde{\alpha})} .
$$

Elasticity $\tilde{\alpha}<\alpha$ measures to what extent firm $i$ internalizes the effects of its own pollution on production. In this decentralized setting, Smulders and Gradus (1996) introduce taxes in production and pollution, and subsidies to abatement in order to bring the decentralized equilibrium towards the central planner's solution. Thus, firm's profit can be written as

$$
\left(1-T_{\mathrm{Y}}\right) Y_{i}-r K_{i}-\left(1-T_{\mathrm{A}}\right) A_{i}-T_{\mathrm{P}} P_{i}
$$

where $r$ is the rental price to capital, $T_{\mathrm{A}}$ is an abatement subsidy rate, $T_{\mathrm{P}}$ is a pollution tax rate and $T_{\mathrm{Y}}$ is a value-added tax rate. Under perfect competition, each firm chooses the abatement level as well as the amount of capital in order to maximize its profits. The first-order conditions for optimality are $^{11}$

$$
\begin{aligned}
\tilde{\psi}_{\mathrm{A}} & =\left[B \frac{\left(1-T_{\mathrm{Y}}\right) \tilde{\alpha} \gamma+\gamma \hat{T}_{\mathrm{P}}}{1-T_{\mathrm{A}}}\right]^{\frac{1}{1-\alpha \gamma}}, \\
\tilde{r}\left(\psi_{\mathrm{A}}\right) & =\left[\left(1-T_{\mathrm{Y}}\right)(\beta-\tilde{\alpha} \lambda)-\lambda \hat{T}_{\mathrm{P}}\right] y_{i}\left(\psi_{\mathrm{A}}\right),
\end{aligned}
$$

where $\hat{T}_{\mathrm{P}}=T_{\mathrm{P}} P_{i} / Y_{i}$. Note that the behavior of firms is independent of consumers' discounting. Therefore, the value of $\psi_{\mathrm{A}}$, as a function of taxes and parameters, is the same as in the case of constant discounting.

In a pure market economy with $T_{\mathrm{Y}}=T_{\mathrm{P}}=T_{\mathrm{A}}=0$, the propensity to abate is lower than in a centralized solution. This is straightforward from (29), which defines a market propensity to abate, $(\tilde{\alpha} \gamma B)^{1 /(1-\alpha \gamma)}$, lower than the minimum feasible propensity to abate in the centralized economy, $(\alpha \gamma B)^{1 /(1-\alpha \gamma)}$ in (13). As pointed out by Smulders and Gradus (1996), in the decentralized scenario producers disregard to some extent the effect of abatement on production as well as the effect of pollution on consumers' utility. The gap in the propensities to abate can be narrowed by the subsidies to abatement or the tax on pollution, but it is contrarily widened with the tax on production.

\footnotetext{
${ }^{11} \mathrm{~A}$ tilde refers to a result in the market economy as opposed to the central planner's solution.
} 
Since all firms are identical, they take the same decisions and, being of measure one, $K=\int_{0}^{1} K_{i} d i=K_{i}$ and $A=\int_{0}^{1} A_{i} d i=A_{i}$. Consequently, $P=P_{i}=A^{-\gamma} K^{\lambda}$, and the aggregate production function reads $Y(t)=\int_{0}^{1} Y_{i}(t) d i=B K^{\beta} P^{-\alpha}$, as given in (1).

The economy is populated by a large number of identical consumers of measure one. The representative $n$-th consumer, $n \in[0,1]$ at each $t$, plays a game against his future selves similar to the one described for the central planner. Because abatement is settled by firms, consumers only choose consumption assuming that the future selves will follow a stationary linear consumption strategy:

$$
\begin{array}{ll} 
& \max _{c_{n t}} \int_{t}^{\infty} U\left(c_{n t}(\tau), P_{t}(\tau)\right) \theta(\tau-t) d \tau, \\
\text { s.t.: } \quad & \dot{k}_{n t}(\tau)=r k_{n t}(\tau)-c_{n t}(\tau), \quad k_{n t}(t)=k_{n t}, \\
& c_{n t}(\tau)=\psi_{\mathrm{C}} k_{n t}(\tau), \quad \tau \geq t .
\end{array}
$$

where $k_{n t}(\tau)$ denotes the accumulated capital for the $n$-th consumer, and $r$ denotes the market interest rate. Note that with no labor, consumers wealth is exclusively determined by the capital stock they own and lend to firms.

For a given value of $\psi_{\mathrm{A}}$ the rate of return in (30) remains constant (like the marginal productivity of capital) provided that $\hat{T}_{\mathrm{P}}$ is also constant. This occurs when the tax on pollution increases at the same speed as the ratio pollution per unit of output decreases. In consequence, under this assumption, for a fixed value of $\psi_{\mathrm{A}}$, the solution to the intergenerational game (31)-(33) would define a balanced growth path.

For these sophisticated (time-consistent) consumers, the optimal growth rate of consumption along a balanced path equilibrium is governed by a modified Ramsey rule, similar to that obtained for the central planner's scenario.

Proposition 8 For $\sigma \leq 1$, the growth rate of a market economy along a balanced path equilibrium for problem (31)-(33) must satisfy the modified Ramsey rule:

$$
\tilde{g}=\frac{r-\Delta(\tilde{g}, \eta)}{1+\eta}
$$

where $\eta$ and $\Delta(g, \eta)$ are given by (15) and (16).

Proof. See the Appendix

Correspondingly, for $\sigma=1$, the modified Ramsey rule particularizes as:

$$
\tilde{g}^{1}=r-\hat{\rho} .
$$


Provided that all firms behave identically, the rate of return is given by (30), and the modified Ramsey rule in the decentralized economy reads

$$
\tilde{g}=\frac{\tilde{r}\left(\psi_{\mathrm{A}}\right)-\Delta(\tilde{g}, \eta)}{1+\eta}, \quad \tilde{g}^{1}=\tilde{r}\left(\psi_{\mathrm{A}}\right)-\hat{\rho} .
$$

Correspondingly, the standard Ramsey curves under constant discounting would be

$$
\tilde{g}_{\hat{\rho}}=\frac{\tilde{r}\left(\psi_{\mathrm{A}}\right)-\hat{\rho}}{1+\eta}, \quad \tilde{g}_{\hat{\rho}}^{1}=\tilde{r}\left(\psi_{\mathrm{A}}\right)-\hat{\rho} .
$$

Note that from (30) an increase in abatement monotonously raises the market rate of return $\tilde{r}^{\prime}\left(\psi_{\mathrm{A}}\right)>0$, but it also has an effect on $\Delta(\tilde{g}, \eta)$. Therefore, the marginal effect of a greater propensity to abate on the growth rate implicitly defined by the modified Ramsey rule in (36) is positive, but softer than the corresponding effect in the case of constant discounting. This is shown by applying the implicit function theorem to the expressions in (36), taking into account Lemma 13 and the standard Ramsey curves in (37) under exponential discounting:

$$
0<\frac{d \tilde{g}}{d \psi_{\mathrm{A}}}=\frac{1}{1+\eta_{\frac{\bar{J}}{\Omega(g, \eta)}}} \tilde{r}^{\prime}\left(\psi_{\mathrm{A}}\right)<\frac{d \tilde{g}_{\hat{\rho}}}{d \psi_{\mathrm{A}}}=\frac{1}{1+\eta} \tilde{r}^{\prime}\left(\psi_{\mathrm{A}}\right) .
$$

The upward sloping curves $\tilde{g}$ and $\tilde{g}_{\hat{\rho}}$ are depicted as the dotted red and blue lines in Figures 3 and 4 . Nonetheless, the propensity to abate in the market economy is known and given by $\tilde{\psi}_{\mathrm{A}}$ in (29). Therefore, the equilibrium growth rate is fully characterized by substituting $\psi_{\mathrm{A}}$ by $\tilde{\psi}_{\mathrm{A}}$ in (36) and (37).

Note too that in a pure market economy, the propensity to abate, $\tilde{\psi}_{\mathrm{A}}$, in (29) partic-

ularizes to $(B \tilde{\alpha} \gamma)^{1 /(1-\alpha \gamma)}$. Provided that the firm does not internalize the whole effect of its own pollution on production, $\tilde{\alpha}<\alpha$, and that the propensity to abate in the centralized scenario must exceed $(B \alpha \gamma)^{1 /(1-\alpha \gamma)}$, then from (29) it follows that the propensity to abate in the pure market economy is lower than the central planner's $\tilde{\psi}_{\mathrm{A}}<\psi_{\mathrm{A}}$. In contrast, the growth rate of the market economy can be above or below the growth rate in the centralized economy.

\section{Comparing social welfare in the centralized and the market economies}

Under non-constant discounting, a sophisticated central planner can be interpreted, as already mentioned, as a representative consumer who internalizes the external effects 
associated with pollution. He takes consumption and abatement decisions to maximize discounted welfare and at the same time, due to the lack of commitment, he must also induce his future selves to behave in his best interest. Consumers in a market economy play a similar intergenerational game with their future selves. As this section will show, contrary to the case of exponential discounting, when individuals present a decreasing degree of impatience the central planner's solution does not necessarily lead to higher social welfare. This result is in line with Krusell et al. (2002), although the underlying mechanism is different. In the Neoclassical growth model proposed in Krusell et al. (2002), the central planner acknowledges that he affects the returns to savings, while the price-taker individuals in a decentralized setting perceive these returns as constant. Thus, a central planner approaching the steady-state equilibrium from below has a decreasing marginal propensity to save, while it is constant for the competitive agent. In consequence, the latter saves more and the competitive economy grows faster, allowing for a higher welfare. In contrast, this section presents a similar result, but for an endogenous growth model and based on different grounds. In particular, two conditions are needed: a strong externality of pollution on utility, which induces the central planner to slow down growth, and a strong decay in the instantaneous discount rate, that is, strongly long-run oriented agents.

When comparing the growth rates in the market and the centralized economies, we can also distinguish the two possible situations commented on by Smulders and Gradus (1996). If the representative firm internalizes a small part of the effect of its emissions on production, (i.e. $\tilde{\alpha}$ and hence $\tilde{\psi}_{\mathrm{A}}$ are small), then the central planner's solution characterized by $\psi_{\mathrm{A}}^{*}>\tilde{\psi}_{\mathrm{A}}$ will almost certainly lead to a faster growth, as displayed by equilibria $E$ and $\tilde{E}$ in Figure 3. In contrast, if the firm internalizes a large part of its externality on production and, at the same time, pollution has a strong impact on utility, (i.e. large $\phi$ ), then the market equilibrium would be characterized by high abatement efforts and fast growth, while the centralized solution, in which the strong externality on utility is internalized, would be associated with a slower growth. This situation is shown in Figure 4.

Insert Figures 3 and 4 about here

If we restrict our analysis to the particular case with log-utility $(\sigma=1)$, the growth rate of the economy is independent of whether individuals discount the future at a constant rate, $\hat{\rho}$, or at a decreasing rate (as long as the same overall impatience is 
assumed). These rates in the centralized and the market economy read:

$$
\begin{aligned}
& g_{\hat{\rho}}^{1 *}=g^{1 *}=r\left(\psi_{\mathrm{A}}^{1 *}\right)-\hat{\rho}=\beta y\left(\psi_{\mathrm{A}}^{1 *}\right)-\psi_{\mathrm{A}}^{1 *} \frac{\lambda}{\gamma}-\hat{\rho} \quad \text { with } \psi_{\mathrm{A}}^{1 *}=\psi_{\mathrm{A} \hat{\rho}}^{1 *}>(\tilde{\alpha} \gamma B)^{\frac{1}{1-\alpha \gamma}}(39 \\
& \tilde{g}_{\hat{\rho}}^{1 *}=\tilde{g}^{1 *}=\tilde{r}\left(\tilde{\psi}_{\mathrm{A}}\right)-\hat{\rho}=(\beta-\tilde{\alpha} \lambda) y\left(\tilde{\psi}_{\mathrm{A}}\right)-\hat{\rho} \quad \text { with } \tilde{\psi}_{\mathrm{A}}=\tilde{\psi}_{\mathrm{A} \hat{\rho}}=(\tilde{\alpha} \gamma B)^{\frac{1}{1-\alpha \gamma}}
\end{aligned}
$$

In these circumstances, the following proposition proves that although the growth rate is identical, individuals with non-constant discounting experience higher welfare than standard agents under exponential discounting. This result immediately follows if the growth rate is positive, given that the discounting method values the long run more strongly than exponential discounting, as clearly shown in Figure 1.

Proposition 9 Assuming log-utility $(\sigma=1)$ and $g^{1 *}, \tilde{g}^{1 *}>0$, the consumer welfare is higher in the case of non-constant discounting than under the standard exponential discounting, both for the centrally planned and for the market economy. That is, ${ }^{12}$

$$
\begin{aligned}
& W^{1}\left(K_{t}\right)=W_{\hat{\rho}}^{1}\left(K_{t}\right)+\frac{g^{1 *}[1+\phi(\gamma-\lambda)]}{\hat{\rho}}\left(\bar{J}_{0}-\frac{1}{\hat{\rho}}\right)>W_{\hat{\rho}}^{1}\left(K_{t}\right), \\
& \widetilde{W}^{1}\left(K_{t}\right)=\widetilde{W}_{\hat{\rho}}^{1}\left(K_{t}\right)+\frac{\tilde{g}^{1 *}[1+\phi(\gamma-\lambda)]}{\hat{\rho}}\left(\bar{J}_{0}-\frac{1}{\hat{\rho}}\right)>\widetilde{W}_{\hat{\rho}}^{1}\left(K_{t}\right),
\end{aligned}
$$

with $\bar{J}_{0}=\hat{\rho} \int_{0}^{\infty} j \theta(j) d j>1 / \hat{\rho}$.

Proof. See the Appendix

Under log-utility, despite an identical growth rate as well as the same overall impatience, agents who discount the future at a decreasing rate assign a higher weight to the long run. In consequence, with positive growth they attain higher welfare. The gap $\bar{J}_{0}-1 / \hat{\rho}$ in (41) and (42) measures how different from exponential discounting consumers' time preferences are. Under constant discounting $\bar{J}_{0}-1 / \hat{\rho}=0$, and the gap widens with the speed of decay of the instantaneous discount rates with the time distance from the present.

If economic growth in the central planner's solution is faster than or equally rapid as in the market economy $\left(g^{1 *} \geq \tilde{g}^{1 *}\right.$ as in Figure 3$)$ then, since the central planner's solution is Pareto optimal under constant discounting, equations (41) and (42) imply that the central planner's solution is also Pareto optimal under non-constant discounting:

$$
W^{1}\left(K_{t}\right)-\widetilde{W}^{1}\left(K_{t}\right) \geq W_{\hat{\rho}}^{1}\left(K_{t}\right)-\widetilde{W}_{\hat{\rho}}^{1}\left(K_{t}\right) \geq 0
$$

\footnotetext{
${ }^{12}$ Because all consumers are identical, $k_{n t}=k_{t}=\int_{0}^{1} k_{t} d n=K_{t}$. Thus, in short notation, $\widetilde{W}^{1}\left(k_{n t}, K_{t}\right)$ and $\widetilde{W}_{\hat{\rho}}^{1}\left(k_{n t}, K_{t}\right)$ are simply denoted as $\widetilde{W}^{1}\left(K_{t}\right)$ and $\widetilde{W}_{\hat{\rho}}^{1}\left(K_{t}\right)$.
} 
However, if the market economy grows faster $\left(\tilde{g}^{1 *}>g^{1 *}\right.$ as in Figure 4), next proposition shows that, contrary to the case of exponential discounting, if individuals discount the future at a non-constant rate they could, under certain condition, derive higher welfare in the market equilibrium than in the central-planner solution.

Proposition 10 Assuming log-utility $(\sigma=1)$, if the growth rate in the market economy is higher than the growth rate in the central-planner solution, $\tilde{g}^{1 *}>g^{1 *}$, then $\widetilde{W}^{1}\left(K_{t}\right)>$ $W^{1}\left(K_{t}\right)$ if and only if

$$
\bar{J}_{0}>\frac{1}{\hat{\rho}}-\hat{\rho} \frac{W_{\hat{\rho}}^{1}\left(K_{t}\right)-\widetilde{W}_{\hat{\rho}}^{1}\left(K_{t}\right)}{\left(g^{1 *}-\tilde{g}^{1 *}\right)(1+\phi(\gamma-\lambda))} .
$$

Proof. The proof is straightforward from the expressions of $\widetilde{W}^{1}\left(K_{t}\right)$ and $W^{1}\left(K_{t}\right)$ in (41) and (42).

Under log-utility, $g^{1 *}=g_{\hat{\rho}}^{1 *}$ and $\tilde{g}^{1 *}=\tilde{g}_{\hat{\rho}}^{1 *}$ and, in consequence, the RHS in condition (43) is invariant to considering constant or non-constant discounting. ${ }^{13}$ In contrast, the LHS of this inequation, $\bar{J}_{0}$, which can be interpreted as the mean of the probability distribution $\omega_{0}(j)=\theta(j) / \int_{0}^{\infty} \theta(i) d i=\hat{\rho} \theta(j)$, can take a value as large as is needed by considering a sufficiently fast decay in the instantaneous discount rate is. Thus, there always exists a sufficiently fast speed of decay in the instantaneous discount rate above which condition (43) is satisfied.

Proposition 10 states that the balanced path equilibrium associated with a decentralized economy can provide a higher social welfare than the central planner's solution under two conditions. It is first necessary that competitive agents save more and grow faster than the central planner. As already pointed out by Smulders and Gradus (1996) this is not based on the way individuals discount the future, but on the relative size of the pollution externalities. If the pollution externality on utility is relatively large, with respect to the pollution externality on production, the central planner would prefer a slower growth rate along the balanced path. Secondly, individuals' instantaneous discount rate should decrease sharply with the time distance from the present (condition (43)), implying that they value the future more strongly, and hence, can attain a higher social welfare in a decentralized economy which grows indefinitely at a faster rate.

\footnotetext{
${ }^{13}$ Indeed, the gap $W_{\hat{\rho}}^{1}\left(K_{t}\right)-\widetilde{W}_{\hat{\rho}}^{1}\left(K_{t}\right)$ is not even dependent on the actual stock of capital, $K_{t}$, as shown in the proof of Proposition 9 in the Appendix.
} 
Example 11 To illustrate the previous result we assume a hybrid exponential-hyperbolic discounting as in Tsoukis et al. (2017), that is,

$$
\theta(j)=(1+\delta j)^{-\varphi / \delta} e^{-\rho j}
$$

where $\rho>0,0<\varphi<1, \delta>0$ and $\varphi / \delta<1$. The instantaneous discount rate $\rho(j)=\rho+\varphi /(1+\delta j)$ decreases with $j$. Note that if $\varphi$ tends to 0 , then the discount function converges to exponential discounting. Applying the properties of the Laplace transformation to (44) as in Dyke (2014), the value of $\bar{J}_{0}$ follows:

$$
\bar{J}_{0}=\frac{1-\varphi / \delta}{\rho}+\frac{1}{\delta}\left(\frac{\hat{\rho}}{\rho}-1\right)
$$

The simulations have been carried out considering the following benchmark parameters' values: $\hat{\rho}=.05, \beta=.9, \lambda=.8, \gamma=1, \alpha=.5, B=.55, \tilde{\alpha}=0.4, \phi=2$. For these parameters, as long as the overall impatience remains equal to .05, it holds that $\tilde{g}^{1 *}>g^{1 *}$. Our simulations illustrate the conditions under which this inequality also implies a Pareto improving market solution. That is, which values of the triplet $(\rho, \varphi, \delta)$ fulfill condition (43). Note that for any pair $\rho$ and $\varphi$, the value of $\delta$ is determined by the assumption of identical overall level of impatience (18). Following the reasoning in Tsoukis et al. (2017) this assumption can be written as:

$$
\hat{\rho}=\frac{e^{-\rho / \delta} \delta^{\varphi / \delta} \rho^{1-\varphi / \delta}}{\Gamma(1-\varphi / \delta, \rho / \delta)}
$$

where $\Gamma(a, b)=\int_{b}^{\infty} x^{a-1} e^{-x} d x$ is the incomplete Gamma function. ${ }^{14}$

In Figures 5-8 the shaded areas display the regions within the plane $\rho-\varphi$ at which condition (43) is satisfied, which guarantees a higher social welfare under the decentralized solution. From (44) it follows that the lower is $\rho$ the less important is the exponential part in the definition of the discount function. Likewise a higher $\varphi$ corresponds to a wider range of variation for the instantaneous discount rate $\rho(j)$. Both a lower $\rho$ or a larger $\varphi$ implies a discount function more distant from exponential discounting, meaning a strong decay in the instantaneous discount rate in the near future. The shadow area in Figures 5-8 shows that the market leads to higher social welfare provided that either $\rho$ is small or $\varphi$ is large, i.e. if the discount is sufficiently different from constant discounting. For the parameters values above, in the particular case with $\rho=1.2 \times 10^{-3}$ and $\varphi=0.9$ (for which $\delta=1.39$ under the assumption of identical overall impatience), the first row

\footnotetext{
${ }^{14}$ We have corrected for a mistake in the expression proposed by Tsoukis et al. (2017), changing the standard Gamma function by an incomplete Gamma function.
} 
in Table 1 shows that the market economy grows faster and provides a higher social welfare than the centralized economy, $W^{1}-\widetilde{W}^{1}<0$. This is in contrast with the case of exponential discounting, with the standard result of a Pareto superior planner solution, $W_{\hat{\rho}}^{1}-\widetilde{W}_{\hat{\rho}}^{1}>0$.

Next, we carry out a sensitivity analysis to analyze the role of the two externalities. An increment in $\tilde{\alpha}$ represents a reduction in the environmental externality on production, $\alpha-\tilde{\alpha}$. Correspondingly an increment in $\phi$ represents a higher environmental externality on utility, or equivalently more environmentally concerned consumers. As shown in the second and third rows in Table 1, the higher the externality on utility with respect to the externality on production, the faster the growth in the market with respect to the centralized solution. As the gap between these growth rates widens, also the social welfare surplus in the market economy under non-constant discounting widens. Moreover, Figures 5 and 6 show that the area consistent with a Pareto improving market solution also expands as the externality on utility increases with respect to the externality on production.

Finally, we analyze how our result is affected by the effectiveness of abatement, $\gamma$, and the effect of capital on pollution, $\lambda$. Note that, under (3) we control the experiment to guarantee the existence of a balanced path equilibrium, hence a reduction in $\lambda$ or an increment in $\gamma$ comes together with a reduction in the output elasticity of production, $\alpha$. That is, since abatement is proportional to capital, the less pronounced the effect of growth on pollution the stronger must be the effect of pollution on output. As shown in the last two rows of Table 1, both lower pollution consequences of capital or higher effectiveness of abatement induce a market social welfare well above the centralized solution when agents discount the future at non-constant rates. Note that this is true even though in the first case the gap in growth rates shrinks, while in the second it expands. Again, Figures 7 and 8 show that the region at which welfare in the market is higher than in the centralized economy becomes wider with either a lower $\lambda$ or a higher $\gamma$.

Insert Table 1 about here

Insert Figures 5 and 6 about here Insert Figures 7 and 8 about here

The counterintuitive result that the competitive economy can provide a higher social welfare than the central planer solution is obtained for the particular case of a log-utility. The hypothesis of an elasticity of intertemporal substitution equal to one is made for simplicity. For the more general case of $\sigma<1$, the analysis is more cumbersome. A 
condition similar to (43) can be found. Whether this condition can or cannot be satisfied remains to be solved both analytically or numerically. At most it can be conjectured that the main result presented in Proposition 10 also holds for $\sigma$ sufficiently close to one. In fact, in a parallel research, for a simpler model with only an externality in utility, it has been possible to analytically prove a similar result for $\sigma$ different from one (see, Cabo et al. 2019).

As stated in Proposition 10, for a log-utility, if the externality on utility is large in relative terms to the production externality, and if discounting highly differs from exponential discounting, then the market economy is welfare enhancing. In consequence, policy measures are inappropriate.

For the general case of an isoelastic utility with $\sigma<1$, it might be pertinent to move the market equilibrium towards the planner's solution. As stated by Smulders and Gradus (1996), this gap can be closed by using either a single pollution tax, $\hat{T}_{\mathrm{P}}$, or a combination of an income tax, $T_{\mathrm{Y}}$, together with an abatement subsidy, $T_{\mathrm{A}}$. The immediate question that arises is whether these policies are more or less effective under non-constant than under constant discounting. As Figures 3 and 4 show, the gap between the centralized and the decentralized growth rates is not the same if individuals discount at a constant rate or their discount rate decreases with the time distance from the present. Thus, to analyze effectiveness, the next proposition computes and compares the elasticity of this gap for non-constant and for constant discounting to changes in either a tax or the subsidy.

Proposition 12 For $\sigma<1$ the elasticity of the gap between the growth rates under a central planner and the market economy with no taxes (NT), with respect to increments in any of the taxes/subsidy is lower under non-constant discounting than in the standard case of exponential discounting, that is,

$$
\left|\frac{d\left(g^{*}-\tilde{g}^{N T *}\right)}{d T} \frac{T}{g^{*}-\tilde{g}^{N T *}}\right|<\left|\frac{d\left(g_{\hat{\rho}}^{*}-\tilde{g}_{\hat{\rho}}^{N T *}\right)}{d T} \frac{T}{g_{\hat{\rho}}^{*}-\tilde{g}_{\hat{\rho}}^{N T *}}\right|, \quad \text { with } T \in\left\{T_{A}, T_{Y}, \hat{T}_{P}\right\},
$$

with $\tilde{g}^{N T *}$ and $\tilde{g}_{\hat{\rho}}^{N T *}$ the equilibrium growth rates of the market economy when $T_{Y}=\hat{T}_{P}=$ $T_{A}=0$, under non-constant and constant discounting respectively.

In the particular case of $\sigma=1$ the elasticity is the same in the two scenarios.

Proof. See the Appendix

In the case of an iso-elastic utility with $\sigma<1$, this proposition states that the imposition of taxes/subsidy is less effective in narrowing the absolute gap in growth rates 
when individuals discount at a non-constant rate. More properly, this lower effectiveness refers to a marginal rise starting from an initial situation of no taxes/subsidy in the market economy. Nevertheless, this does not imply a lower effectiveness when raising the tax/subsidy from an already positive level. By contrast, in the particular case $\sigma=1$, when the central planner solution is welfare enhancing, the policy measures are equally effective with constant and non-constant discounting.

\section{Conclusions}

This paper introduces non-constant discounting within the framework of the model of pollution abatement and endogenous growth presented by Smulders and Gradus (1996). In this model pollution is a by-product of production and can be abated. It is associated with two externalities, negatively affecting both the utility of consumers and the marginal productivity of capital. Within this context, the optimal consumption and abatement decision, when taken by dynamic individuals, will be dependent on whether their rate of time preference remains constant or decreases with the time distance from the present.

Under the standard assumption of exponential discounting decisions taken at a given point in time will continue to be optimal if recomputed at any later time. The time consistency of the solution implies that the central planner's solution coincides with the social optimum. Therefore, under the hypothesis of constant discounting and regardless of whether the market economy is characterized by lower/higher growth, bringing the market equilibrium towards the central-planner equilibrium is welfare enhancing. It may be, then, appropriate to impose taxes or subsidies to move the decentralized economy towards the social optimum. This is not necessarily the case if individuals discount the future at a decreasing rate.

When dealing with non-constant discounting, we have made two important assumptions. First, we neglect the possibility of commitment, and consider that individuals are time-consistent sophisticated agents. They know that their future selves will not stick to current planned decisions, and to prevent this time inconsistency, they play a game against their future selves restricted to the assumption that all future cohorts will play the same linear strategies. Under stationarity the Markov perfect strategies played by future selves coincide with the anticipated decisions and hence constitute an equilibrium. This sophisticated behavior is followed by competitive agents in the decentralized solution, and also by the central planner. Given the lack of commitment, the central planner 
can be interpreted as a representative consumer who internalizes the two externalities caused by pollution, and takes consumption and abatement decisions as the solution to the intergenerational game.

Second, the analysis is controlled to have an identical overall level of impatience. This assumption guarantees that the comparison of the results under constant and nonconstant discounting depends only on the way individuals discount the future, and not on different degrees of impatience. From this assumption it immediately follows that the individuals with a decreasing rate of impatience value relatively softly the short run and relatively strongly the long run, compared to standard agents with constant discounting. With positive growth and an elasticity of intertemporal substitution lower than one, current increments in consumption provoke increments in current and ulterior utility, although the marginal effect weakens with the time distance from the present. In contrast, the marginal effect on ongoing utility is constant under exponential discounting. Therefore, individuals with non-constant discounting undervalue the effect of current savings on ongoing utility, and hence invest less. This leads to our first result: under the empirically relevant hypothesis of an elasticity of intertemporal substitution lower than one, the fact that the households discount the future at a decreasing rate leads to a balanced growth path characterized by a lower growth rate and higher abatement efforts. In contrast, with log-utility the equilibrium remains unchanged regardless of whether we consider time-declining or constant discounting.

Our main result lies in the comparison of social welfares in the centralized and the decentralized economy under non-constant discounting. Having no commitment power, the planner's solution stemming from the game between subsequent generations does not necessarily reach the social optimum. More importantly, we prove that the social welfare in the market economy surpasses the central planner's solution when certain conditions are met. This is consistent with the symmetric result in Krusell et al. (2002) for a Neoclassical growth model, although the underlying reasoning diverges. A central planner who internalizes the two externalities caused by pollution might be inclined to reduce the growth rate of the economy. As already stated by Smulders and Gradus (1996) for the case with constant discounting, this can occur when the externality of pollution on utility is relatively strong with respect to the externality on production. A faster growth is welcomed by those individuals who discount the future at a non-constant rate, and value the short run softer and the long run stronger than the standard individuals with constant discounting. Thus, for log-utility, and if the market economy grows faster 
than the centralized economy, we prove that this faster growth leads to a higher social welfare if the individuals' degree of impatience decays rapidly with the time distance from the present, and hence, the value assigned by current individuals to the distant future is very high. The result is valid for a general discount function satisfying a continuous decay in the instantaneous discount rate. A numerical example is presented for a specific discount function to illustrate this result. This example highlights that the more individuals' preferences separate from constant discounting (either due to a faster or a deeper decay in the instantaneous discount rate) the more easily this result emerges.

For an economic growth model with environmental externalities, two main policy implications arise when indivivuals discount at a decreasing rate. In the case of a logutility, internalizing the externalities caused by pollution might not be welfare improving. Therefore, taxes or subsidies to approach the central planner solution would not be appropriate. On the other hand, if the elasticity of intertemporal substitution is lower than one, and if the decentralized market economy does not welfare dominate the central planner, then being appropriate to impose taxes on pollution or grant subsidies on abatement, these policies are less effective than under constant discounting. This has been proven when the policies are introduced starting from an initial situation of no intervention.

\section{References}

[1] Andersen, S., Harrison, G.W., Morten, I.L. and Rutström, E.E. (2014). Discounting behavior: a reconsideration. European Economic Review 71, 15-33.

[2] Barro, R.J. (1999). Ramsey meets Laibson in the Neoclassical growth model. Quarterly Journal of Economics 114, 1125-1152.

[3] Barro, R. and Sala-i-Martin, X. (2004), Economic Growth. second ed., MIT Press Cambridge, Massachusetts.

[4] Cabo, F., Martín-Herrán, G. and Martínez-García, M.P. (2015). Non-constant discounting and Ak-type growth models. Economics Letters 131, 54-58.

[5] Cabo, F., Martín-Herrán, G. and Martínez-García, M.P. (2016). Unbounded growth in the Neoclassical growth model with non-constant discounting. Mathematical Social Sciences 84, 93-104. 
[6] Cabo, F., Martín-Herrán, G. and Martínez-García, M.P. (2019). Present bias and the inefficiency of the centralized economy. The role of the elasticity of intertemporal substitution. Cahier du GERAD G-2019-34.

[7] Caliendo, F.N. and Aadland, D. (2007). Short-term planning and the life-cycle consumption puzzle. Journal of Economic Dynamics and Control 31, 1392-1415.

[8] Castañer, A., Marín-Solano, J. and Ribas, C. (2016). Pareto inefficiency and dynamic bargaining in common property resource games with asymmetric players. www.bioecon-network.org/pages/18th_2016/Marin-Solano.pdf.

[9] Chichilnisky, G. (1996). An axiomatic approach to sustainable development, Social Choice and Welfare 13, 231-257.

[10] DellaVigna, S. (2009). Psychology and economics: Evidence from the field. Journal of Economic Literature 47, 315-372.

[11] Dyke, P. (2014), An Introduction to Laplace Transforms and Fourier Series, Springer, London, UK.

[12] Farzin, Y.H. and Wendner, R. (2014). The time path of the saving rate: hyperbolic discounting and short-term planning. MPRA Paper No. 54614, posted 19. March 2014.

[13] Findley, T.S. and Caliendo, F.N. (2014). Interacting mechanisms of time inconsistency. Journal of Economics Psychology 41, 68-76.

[14] Frederick, S., Loewnstein, G. and O’Donoghue, T. (2002). Time discounting and time preference: a critical review. Journal of Economics Literature 40, 351-401.

[15] Fujii, T and Karp, L. (2008). Numerical analysis of non-constant pure rate of time preference: a model of climate policy. Journal of Environmental Economics and Management 56, 83-101.

[16] Gerlagh, R and Liski, M. (2017). Climate policies with non-constant discounting. Journal of the European Economic Association doi: 10.1093/jeea/jvx010

[17] Goldman, S.M. (1980). Consistent plans. Review of Economic Studies 47, 533-537. 
[18] Groom, B., Hepburn, C., Koundouri, P. and Pearce, D. (2005). Declining Discount Rates: The Long and the Short of it. Environmental and Resource Economics 32, 445-493.

[19] Hepburn, C., Duncan, S. and Papachristodoulou, A. (2010) Behavioural economics, hyperbolic discounting and environmental policy. Environmental and Resource Economics 46, 189-206.

[20] Hiraguchi, R. (2014). On the neoclassical growth model with non-constant discounting. Economics Letters 125, 175-178.

[21] Hiraguchi, R. (2016). On a two-sector endogenous growth model with quasigeometric discounting. Journal of Mathematical Economics 65, 26-35.

[22] Karp, L. (2005). Global warming and hyperbolic discounting. Journal of Public Economics, 89, 261-282.

[23] Karp, L. (2007). Non-constant discounting in continuous time. Journal of Economic Theory, 132, 557-568.

[24] Karp, L. and Tsur, Y. (2011). Time perspective and climate change policy. Journal of Environmental Economics \&3 Management 62, 1-14.

[25] Krusell, P., Kuruş̧̧u, B. and Smith, A. A. Jr. (2002). Equilibrium welfare and government policy with quasi-geometric discounting. Journal of Economic Theory $105,42-72$.

[26] Laibson, D. (1997). Golden eggs and hyperbolic discounting. Quarterly Journal of Economics 112, 443-477.

[27] Leininger, W. (1986). The Existence of Perfect Equilibria in a Model of Growth with Altruism between Generations. Review of Economic Studies 53, 349-67.

[28] Li, C.Z. and Lofgren, K.G. (2000). A dynamic analysis with heterogeneous time preferences. Journal of Environmental Economics 83 Management, 40, 236-250.

[29] Marín-Solano, J. (2015). Group inefficiency in a common property resource game with asymmetric players. Economic Letters 136, 214-217.

[30] Marín-Solano, J. and Navas, J. (2009). Non-constant discounting in finite horizon: the free terminal time case. Journal of Economic Dynamics \& Control 33, 666-675. 
[31] Phelps, E.S., and Pollak, R.A. (1968). On Second-Best National Saving and GameEquilibrium Growth. The Review of Economic Studies 35, 85-19.

[32] Pollak, R.A. (1968). Consistent Planning. Review of Economic Studies 35, 201-208.

[33] Ramsey, F. (1928). A mathematical theory of saving. Economic Journal 38, 543559.

[34] Samuelson, P.A. (1937). A note on measurement of utility. Review of Economic Studies 4, 155-161.

[35] Smulders, S. and Gradus, R. (1996). Pollution abatement and long-term growth. European Journal of Political Economy 12, 505-532.

[36] Sorger, G. (2004). Consistent planning under quasi-geometric discounting. Journal of Economic Theory 118, 118-129.

[37] Strotz, R.H. (1956). Myopia and inconsistency in dynamic utility maximization. Review of Economic Studies 23, 165-80.

[38] Strulik, H. (2015). Hyperbolic discounting and endogenous growth. Economics Letters $126,131-134$.

[39] Tsoukis, C., Tournemaine, F. and Gillman, M. (2017). Hybrid exponentialhyperbolic discounting and growth without commitment. The Manchester School, doi:10.1111/manc.12197. 


\section{A Appendix}

\section{Proof of Proposition 1.}

Isoelastic utility with $\sigma<1$

For linear strategies, given the utility function and the dynamics of the capital stock we conjecture a value function of the form:

$$
W\left(K_{t}\right)=W_{0}+W_{1} K_{t}^{-\eta}
$$

with $W_{0}, W_{1}$ and $\eta$ unknowns to be determined. From (12) and (45), the first-order condition (10) reads

$$
\left\{C_{t}(\tau)\left[K_{t}(\tau)^{\lambda}\left(\psi_{\mathrm{A}} K_{t}(\tau)\right)^{-\gamma}\right]^{-\phi}\right\}^{1-\frac{1}{\sigma}}=-C_{t}(\tau) \eta W_{1} K_{t}(\tau)^{-\eta-1}
$$

For consumption to be proportional to capital, the value of $\eta$ must be as defined in (15). Constant $W_{0}$ can be derived from the definition of the value function, conjecture (45) and the overall impatience in (18):

$$
W_{0}=\frac{\sigma}{(1-\sigma) \hat{\rho}} .
$$

Likewise, constant $W_{1}$ can be obtained from (46):

$$
W_{1}=-\frac{\psi_{\mathrm{C}}^{-\frac{1}{\sigma}} \psi_{\mathrm{A}}^{-\frac{1-\sigma}{\sigma} \phi \gamma}}{\eta} .
$$

where the relation between $\psi_{\mathrm{C}}$ and $\psi_{\mathrm{A}}$ is given in (12).

From the optimality conditions and the conjectured value function, the RHS of the Bellman equation in (9) along the balanced path can be rewritten as

$$
\frac{\sigma}{1-\sigma}-\eta W_{1}\left(g-\frac{\sigma}{1-\sigma} \psi_{\mathrm{C}}\right) K_{t}^{-\eta}
$$

On the other hand, from the definition of $\rho(j)$ it is immediately obvious that $\int_{t}^{\infty} \rho(\tau-$ t) $\theta(\tau-t) d s=1$, and, therefore, the LHS of the Bellman equation can be written as

$$
\frac{\sigma}{1-\sigma}+\frac{\sigma \psi_{\mathrm{C}} \eta W_{1}}{1-\sigma} \int_{t}^{\infty} K_{t}(\tau)^{-\eta} \theta(\tau-t) \rho(\tau-t) d \tau
$$

Along a balanced growth path, the capital stock at the running time $\tau$, for the $t$-agent is $K_{t}(\tau)=K_{t} e^{g(\tau-t)}$. Therefore, equating expressions (49) and (50) it follows:

$$
-\frac{\sigma \psi_{\mathrm{C}}}{1-\sigma} \int_{t}^{\infty} e^{-\eta g(\tau-t)} \theta(\tau-t) \rho(\tau-t) d \tau=g-\frac{\sigma \psi_{\mathrm{C}}}{1-\sigma}
$$


The assumption $\sigma<1$ implies $\eta>0$ and, hence, it guarantees the convergence of this integral when the growth rate of the economy is a positive constant. Integrating by parts one gets

$$
\int_{t}^{\infty} e^{-\eta g(\tau-t)} \theta(\tau-t) \rho(\tau-t) d \tau=1-\eta g \int_{t}^{\infty} e^{-g \eta(\tau-t)} \theta(\tau-t) d \tau .
$$

And from (51) and (52) it follows:

$$
\frac{\int_{t}^{\infty} e^{-g \eta(\tau-t)} \theta(\tau-t) \rho(\tau-t) d \tau}{\int_{t}^{\infty} e^{-g \eta(\tau-t)} \theta(\tau-t) d \tau}=\left(\frac{\sigma \psi_{\mathrm{c}}}{1-\sigma}-g\right) \eta
$$

The ratio of integrals in the LHS defines the effective rate of time preference, $\Delta(g, \eta)$, in (16). Then,

$$
\Delta(g, \eta)=\left(\frac{\sigma \psi_{\mathrm{C}}}{1-\sigma}-g\right) \eta
$$

By adding and subtracting $g=y\left(\psi_{\mathrm{A}}\right)-\psi_{\mathrm{C}}-\psi_{\mathrm{A}}$ in the RHS, and taking into account the constant returns to scale hypothesis in (3) together with the consumption-abatement ratio in (12) and the expression for $\eta$ in (15), equation (53) can be rewritten as

$$
\Delta(g, \eta)=\beta y\left(\psi_{\mathrm{A}}\right)-\frac{\lambda}{\gamma} \psi_{\mathrm{A}}-(1+\eta) g
$$

which leads to the Ramsey equation in (14).

Logarithmic utility: $\sigma=1$

The value function along a balanced growth path can be expressed as

$$
\begin{aligned}
W^{1}\left(K_{t}\right)= & \int_{t}^{\infty} \ln \left[C_{t} P_{t}^{-\phi} e^{g^{1}[1+\phi(\gamma-\lambda)](\tau-t)}\right] \theta(\tau-t) d \tau= \\
& \ln \left[C_{t} P_{t}^{-\phi}\right] \int_{t}^{\infty} \theta(\tau-t) d \tau+g^{1}[1+\phi(\gamma-\lambda)] \int_{t}^{\infty}(\tau-t) \theta(\tau-t) d \tau= \\
& \frac{[1+\phi(\gamma-\lambda)]}{\hat{\rho}} \ln K_{t}+\frac{1}{\hat{\rho}} \ln \left(\psi_{\mathrm{C}} \psi_{\mathrm{A}}^{\gamma \phi}\right)+\frac{g^{1} \bar{J}_{0}[1+\phi(\gamma-\lambda)]}{\hat{\rho}}
\end{aligned}
$$

with $\bar{J}_{0}=\hat{\rho} \int_{0}^{\infty} j \theta(j)$. Then, the value function is defined as

$$
W^{1}\left(K_{t}\right)=W_{0}^{1}+W_{1}^{1} \ln K_{t}
$$

with

$$
W_{0}^{1}=\frac{g^{1}[1+\phi(\gamma-\lambda)] \bar{J}_{0}+\ln \left(\psi_{\mathrm{C}} \psi_{\mathrm{A}}^{\gamma \phi}\right)}{\hat{\rho}}, \quad W_{1}^{1}=\frac{1+\phi(\gamma-\lambda)}{\hat{\rho}}
$$


On the other hand, the first-order condition (10) reads

$$
\frac{1}{C_{t}}=\frac{W_{1}^{1}}{K_{t}}
$$

Then, from (7), (12), (56) and (57), the growth rate of the economy can be written as

$$
g^{1}=y\left(\psi_{\mathrm{A}}\right)-\psi_{\mathrm{A}}-\frac{\hat{\rho}}{1+\phi(\gamma-\lambda)}=\beta y\left(\psi_{\mathrm{A}}\right)-\psi_{\mathrm{A}} \frac{\lambda}{\gamma}-\hat{\rho}
$$

Lemma 13 If $\sigma<1$ and $g>0$, when the future is discounted at a non-constant rate, $\Omega(g, \eta)$ in (22) satisfies

$$
\frac{d \Omega}{d g}=-\eta \bar{J} \Omega(g, \eta)<0, \quad \frac{d \Omega}{d \eta}=-g \bar{J} \Omega(g, \eta) \leq 0(<0 \text { if } g>0),
$$

with $\bar{J}=\int_{0}^{\infty} j \omega(j) d j \in(0, \infty), \quad \omega(j)=\frac{\theta(j) e^{-\eta g j}}{\Omega(g, \eta)} \in(0,1)$.

Proposition 14 Under condition $\sigma<1$ and $\hat{\rho}<r\left(\arg \min _{\psi_{A}} r\left(\psi_{A}\right)\right)$, the modified Ramsey rule (14) implicitly defines a positive growth rate, $g$, as a function of the propensity to abate $\psi_{A}$.

\section{Proof.}

Integrating by parts in (16), one gets expression $(60), \Delta(g, \eta)=1 / \Omega(g, \eta)-\eta g$. Replacing this expression into equation (14) and rearranging terms we get

$$
g+\frac{1}{\Omega(g, \eta)}=r\left(\psi_{\mathrm{A}}\right)
$$

Since, from $(58) d \Omega(g, \eta) / d g<0$, the LHS in (59) can be regarded as a monotonously increasing function of $g$. Moreover, $\lim _{g \rightarrow 0} \Omega(g, \eta)=1 / \hat{\rho}$ and $\lim _{g \rightarrow+\infty} \Omega(g, \eta)=0$. Then, the LHS runs from $\hat{\rho}$ towards $\infty$ as $g$ goes from zero to $\infty$. Moreover, for any $\psi_{\mathrm{A}} \in$ $\left((B \alpha \gamma)^{\frac{1}{1-\alpha \gamma}}, B^{\frac{1}{1-\alpha \gamma}}\right], r\left(\psi_{\mathrm{A}}\right)>0$, and hence, under condition $\hat{\rho}<r\left(\arg \min _{\psi_{\mathrm{A}}} r\left(\psi_{\mathrm{A}}\right)\right)$, there always exists a unique $g>0$ satisfying equation (59).

\section{Proof of Proposition 4.}

Integrating by parts in the expression in (16) makes it possible to re-write the effective rate of time preference as the inverse of the present value of the effect of a marginal increment in current utility on all future utilities, diminished by the speed of decay of the marginal effect of current changes on future utility:

$$
\Delta(g, \eta)=\frac{1}{\Omega(g, \eta)}-\eta g .
$$


Results $\Delta(g, \eta)>\hat{\rho}$ and $\Delta(g, 0)=\Delta(0, \eta)=\hat{\rho}$ follow from (23), (24) and (60).

The relationship between the propensity to consume out of wealth, $\psi_{\mathrm{C}}$, and the growth rate can be obtained by plugging (12) in (21) and comparing it against (48) or (56). And similar analysis in the case of exponential discounting.

$$
\psi_{\mathrm{C}}=\frac{1}{[1+\phi(\gamma-\lambda)] \Omega(g, \eta)}, \quad \psi_{\mathrm{C} \hat{\rho}}=\frac{1}{[1+\phi(\gamma-\lambda)] \Omega_{\hat{\rho}}(g, \eta)} .
$$

For a given value of $g$, it straightforwardly follows from (24) and (61) that $\psi_{\mathrm{C}}>\psi_{\mathrm{C} \hat{\rho}}$ for $\sigma<1$ and $\psi_{\mathrm{C}}=\psi_{\mathrm{C} \hat{\rho}}=\hat{\rho} /([1+\phi(\gamma-\lambda)])$ for $\sigma=1$ or $g=0$.

Lemma $15 \operatorname{Cov}(j, \rho(j))=\frac{\Omega(g, \eta)-\bar{J}}{\Omega(g, \eta)}<0$ and therefore, $\Omega(g, \eta)<\bar{J}$.

\section{Proof.}

$$
\begin{gathered}
\operatorname{Cov}(j, \rho(j))=\int_{0}^{\infty} \rho(j) \omega(j)[j-\bar{J}] d j=\int_{0}^{\bar{J}} \rho(j) \omega(j)[j-\bar{J}] d j+\int_{\bar{J}}^{\infty} \rho(j) \omega(j)[j-\bar{J}] d j \\
<\quad \rho(\bar{J}) \int_{0}^{\bar{J}} \omega(j)[j-\bar{J}] d j+\rho(\bar{J}) \int_{\bar{J}}^{\infty} \omega(j)[j-\bar{J}] d j=\rho(\bar{J}) \int_{0}^{\infty} \omega(j)[j-\bar{J}] d j=0 .
\end{gathered}
$$

Moreover, since $\rho(j)=-\dot{\theta}(j) / \theta(j)$, then

$$
\operatorname{Cov}(j, \rho(j))=\frac{-1}{\Omega(g, \eta)} \int_{0}^{\infty} \dot{\theta}(j)(j-\bar{J}) e^{-\eta g j} d j=\frac{-1}{\Omega(g, \eta)}\left\{\int_{0}^{\infty} j \dot{\theta}(j) e^{-\eta g j} d j-\bar{J} \int_{0}^{\infty} \dot{\theta}(j) e^{-\eta g j} d j\right\} .
$$

Integrating by parts, after some calculus it follows that

$$
0>\operatorname{Cov}(j, \rho(j))=\frac{\Omega(g, \eta)-\bar{J}}{\Omega(g, \eta)} .
$$

\section{Proof of Proposition 5.}

Writing the modified Ramsey equation as

$$
g\left(\psi_{\mathrm{A}}\right)=r\left(\psi_{\mathrm{A}}\right)-\frac{1}{\Omega(g, \eta)}
$$

and differentiating implicitly wrt $\psi_{\mathrm{A}}$, taking into account (58) and (19) one gets

$$
g^{\prime}\left(\psi_{\mathrm{A}}\right)=\frac{r^{\prime}\left(\psi_{\mathrm{A}}\right)}{1+\eta \frac{\bar{J}}{\Omega(g, \eta)}}, \quad g_{\hat{\rho}}^{\prime}\left(\psi_{\mathrm{A}}\right)=\frac{r^{\prime}\left(\psi_{\mathrm{A}}\right)}{1+\eta} .
$$

Because $\eta>0$ and, from Lemma $15 \Omega(g, \eta)<\bar{J}$, then the effect of $\psi_{\text {A }}$ on the growth rate in the case of non-constant discounting is softer than under exponential discounting:

$$
\left|g^{\prime}\left(\psi_{\mathrm{A}}\right)\right|<\left|g_{\hat{\rho}}^{\prime}\left(\psi_{\mathrm{A}}\right)\right| \quad \text { for all } \quad \psi_{\mathrm{A}} \geq 0
$$


From (20) the marginal effect of $\psi_{\mathrm{A}}$ on the social rate of return reads

$$
r^{\prime}\left(\psi_{\mathrm{A}}\right)=\alpha \gamma \beta B \psi_{\mathrm{A}}^{\alpha \gamma-1}-\frac{\lambda}{\gamma} .
$$

Therefore, for $\psi_{\mathrm{A}}<\hat{\psi}_{\mathrm{A}}=\left(\alpha \beta \gamma^{2} B / \lambda\right)^{1 /(1-\alpha \gamma)}, r^{\prime}\left(\psi_{\mathrm{A}}\right)>0$; and for $\psi_{\mathrm{A}}>\hat{\psi}_{\mathrm{A}}, r^{\prime}\left(\psi_{\mathrm{A}}\right)<0$. Then, from $(62)$ the propensity to abate $\hat{\psi}_{\mathrm{A}}$ maximizes the modified Ramsey rule both under constant and non-constant discounting.

Taking into account that $\Omega(g, \eta)<\Omega_{\hat{\rho}}(g, \eta)$, then,

$$
g_{\hat{\rho}}\left(\psi_{\mathrm{A}}\right)=\frac{r\left(\psi_{\mathrm{A}}\right)-\hat{\rho}}{1+\eta}=r\left(\psi_{\mathrm{A}}\right)-\frac{1}{\Omega_{\hat{\rho}}(g, \eta)}>r\left(\psi_{\mathrm{A}}\right)-\frac{1}{\Omega(g, \eta)}=g\left(\psi_{\mathrm{A}}\right),
$$

which proves that the modified Ramsey curve under non-constant discounting lies below the standard Ramsey curve under exponential discounting.

\section{Proof of Proposition 6.}

According to Proposition 5, the modified Ramsey curve with non-constant discounting is located below the standard Ramsey curve under exponential discounting. Furthermore, the downward sloping budget constraint (27) is independent of time preferences. Then, at the crossing point, non-constant discounting implies a lower growth rate and a higher propensity to abate (see Figure 2). The positive relation between $\psi_{A}$ and $\psi_{C}$ established in (12) guarantees also a higher propensity to consume.

\section{Proof of Proposition 8.}

Isoelastic utility with $\sigma<1$

The Bellman equation for the optimization problem (31)-(32) of the $n$-th consumer's belonging to the $t$-cohort reads

$$
\begin{aligned}
\int_{t}^{\infty} & U\left(c_{n t}^{*}(\tau), P_{t}\left(K_{t}(\tau)\right)\right) \theta(\tau-t) \rho(\tau-t) d \tau= \\
& \max _{c_{n t}}\left\{U\left(c_{n t}, P_{t}\left(K_{t}\right)\right)+\widetilde{W_{k_{n t}}^{\prime}}\left(k_{n t}, K_{t}\right)\left[r k_{n t}-c_{n t}\right]+\widetilde{W_{K_{t}}^{\prime}}\left(k_{n t}, K_{t}\right) \dot{K}_{t}\right\},
\end{aligned}
$$

where $c_{n t}^{*}(\tau)$ denotes the optimal consumption planned by the $t$-cohort of the $n$-th consumer for the elapsed time $\tau>t$. The pollution stock $P_{t}\left(K_{t}(\tau)\right)$ depends on the abatement carried out by all firms ${ }^{15}$ and the total capital stock accumulated by all the members of the $t$-cohort from $t$ and up to the elapsed time $\tau, K_{t}(\tau)$. For ease of notation, pollution for the $t$-cohort is written as a function of the elapsed time $\tau$ :

$$
P_{t}(\tau)=P_{t}\left(K_{t}(\tau)\right)=\psi_{\mathrm{A}}^{-\gamma} K_{t}(\tau)^{-(\gamma-\lambda)} .
$$

\footnotetext{
${ }^{15}$ Note that $A_{t}(\tau)$ can be written as $\psi_{\mathrm{A}} K_{t}(\tau)$. Since $\tilde{\psi}_{\mathrm{A}}$ is constant from the optimality condition in (29) then, abatement and capital will be proportional in equilibrium.
} 
From (9) it follows that the value function of the $n$-th consumer, $\widetilde{W}\left(k_{n t}, K_{t}\right)$, depends on the capital stock he owns at time $t, k_{n t}$, whose dynamics is determined by this agent's consumption decisions, and also on the global capital stock at this time $t, K_{t}$. While this global capital stock affects the consumer's welfare through its effect on the pollution stock, the $n$-th consumer does not internalize the effect of his capital stock on the global stock, and hence on the ongoing pollution. Therefore his consumption decision does not affect the dynamics of the global capital stock, and the first-order optimality condition for the problem above reads

$$
U_{c_{n t}}^{\prime}\left(c_{n t}, P_{t}\left(K_{t}\right)\right)=\widetilde{W}_{k_{n t}}^{\prime}\left(k_{n t}, K_{t}\right)
$$

Equivalently, at any ulterior time $\tau$, the maximizer of the RHS of equation (63) reads

$$
c_{n t}^{*}(\tau)=P_{t}(\tau)^{(1-\sigma) \phi}\left(\widetilde{W}_{k_{n t}}^{\prime}(\tau)\right)^{-\sigma}
$$

where, for simplicity, $\widetilde{W_{k_{n t}}^{\prime}}(\tau)$ denotes $\widetilde{W}_{k_{n t}}^{\prime}\left(k_{n t}(\tau), K_{t}(\tau)\right)$.

Plugging this optimal consumption into (2), the utility at the optimal trajectory reads

$$
U\left(c_{n t}^{*}(\tau), P_{t}(\tau)\right)=\frac{\sigma}{1-\sigma}\left[1-P_{t}(\tau)^{(1-\sigma) \phi}\left(\widetilde{W}_{k_{n t}}^{\prime}(\tau)\right)^{1-\sigma}\right] .
$$

Plugging these last two expressions into (63) and taking into account $\int_{t}^{\infty} \rho(\tau-t) \theta(\tau-$ $t) d s=1$, the Bellman equation can be rewritten as

$$
\begin{aligned}
& -\int_{t}^{\infty} \frac{\sigma}{1-\sigma} P_{t}(\tau)^{(1-\sigma) \phi}\left(\widetilde{W}_{k_{n t}}^{\prime}(\tau)\right)^{1-\sigma} \theta(\tau-t) \rho(\tau-t) d \tau= \\
& -\frac{\sigma}{1-\sigma} P_{t}(t)^{(1-\sigma) \phi}\left(\widetilde{W}_{k_{n t}}^{\prime}(t)\right)^{1-\sigma}+\widetilde{W}_{k_{n t}}^{\prime}(t)\left[r k_{n t}-P_{t}(t)^{(1-\sigma) \phi}\left(\widetilde{W}_{k_{n t}}^{\prime}(t)\right)^{-\sigma}\right]+\widetilde{W}_{K_{t}}^{\prime}(t) \dot{K}_{t} .
\end{aligned}
$$

Taking into account in (65) that $k_{n t}, K_{t}$ and $c_{n t}(t)$ grow at the same constant rate along the balanced path, we conjecture a value function of the form

$$
\widetilde{W}\left(k_{n t}(\tau), K_{t}(\tau)\right)=\widetilde{W}_{0}+\widetilde{W}_{1} k_{n t}^{\eta_{1}} K_{t}^{\eta_{2}} .
$$

This conjecture is in the same spirit as Krusell et al. (2002), who consider a logarithmic utility in a discrete setting. Plugging this conjecture into (65), parameters $\eta_{1}$ and $\eta_{2}$ satisfy

$$
\eta_{1}=-\frac{1-\sigma}{\sigma}, \quad \eta_{2}=-\frac{1-\sigma}{\sigma} \phi(\gamma-\lambda), \quad \eta_{1}+\eta_{2}=-\eta
$$

Provided that all consumers are identical, the $n$-th and the global capital stock grow at the same constant rate, $\tilde{g}$, along the balanced path. Then, the individual and the global capital stocks at the running time $\tau$ for the $t$-cohort can be written as $k_{n t}(\tau)=$ 
$k_{n t} e^{\tilde{g}(\tau-t)}$ and $K_{t}(\tau)=K_{t} e^{\tilde{g}(\tau-t)}$, respectively. Along the balanced path output, capital and abatement grow at the same rate and, hence, $\psi_{\mathrm{A}}$ and $r$ remain constant. Then, the LHS of (65) can be written

$$
\Theta K_{t}^{(1-\sigma)\left[\eta_{2}-\phi(\gamma-\lambda)\right]} k_{n t}^{\eta_{1}} \int_{t}^{\infty} e^{-\tilde{g} \eta(\tau-t)} \theta(\tau-t) \rho(\tau-t) d \tau
$$

where

$$
\Theta=-\frac{\sigma}{1-\sigma} \psi_{\mathrm{A}}^{-(1-\sigma) \gamma \phi}\left(\eta_{1} \widetilde{W}_{1}\right)^{1-\sigma}
$$

Expression (68) can be equivalently written as

$$
\int_{t}^{\infty} \rho(\tau-t) \frac{\theta(\tau-t) e^{-\tilde{g} \eta(\tau-t)}}{\int_{t}^{\infty} \theta(\tau-t) e^{-\tilde{g} \eta(\tau-t)} d \tau} d \tau \int_{t}^{\infty}-\frac{\sigma}{1-\sigma} P_{t}(\tau)^{(1-\sigma) \phi}\left(\widetilde{W}_{k_{n t}}^{\prime}(\tau)\right)^{1-\sigma} \theta(\tau-t) d \tau
$$

and from (16) and the definition of the value function, the LHS reads

$$
\Delta(\tilde{g}, \eta)\left(\widetilde{W}\left(k_{n t}, K_{t}\right)-\widetilde{W}_{0}\right) \equiv \Delta(\tilde{g}, \eta) \widetilde{W_{1}} k_{n t}^{\eta_{1}} K_{t}^{\eta_{2}}
$$

Similarly, along the balanced path the RHS of the Bellman equation can be rewritten as

$$
-\frac{1}{1-\sigma}\left(\eta_{1} \widetilde{W}_{1}\right)^{1-\sigma} \psi_{\mathrm{A}}^{-(1-\sigma) \gamma \phi} k_{n t}^{\eta_{1}} K_{t}^{\eta_{2}}+\widetilde{W}_{1} k_{n t}^{\eta_{1}} K_{t}^{\eta_{2}}\left\{\eta_{1} r+\tilde{g} \eta_{2}\right\}
$$

Given expressions (69) and (70), we check whether the conjectured value function satisfies the Bellman equation, and compute constants $\widetilde{W}_{0}, \widetilde{W}_{1}$. Constant $\widetilde{W}_{1}$ is given by

$$
\widetilde{W}_{1}=\frac{1}{\eta_{1}}\left(\frac{1}{[\Delta(\tilde{g}, \eta)+\tilde{g} \eta] \psi_{\mathrm{A}}^{(1-\sigma) \gamma \phi}}\right)^{\frac{1}{\sigma}}
$$

Following the same reasoning as in the proof of Proposition $1, \widetilde{W}_{0}=W_{0}$, given in (47):

$$
\widetilde{W}_{0}=\frac{\sigma}{(1-\sigma) \hat{\rho}} .
$$

Finally, to compute the modified Ramsey rule, we take derivatives with respect to $k_{n t}$ in the RHS of (65) and in (69), and obtain

$$
\Delta(\tilde{g}, \eta) \widetilde{W_{k_{n t}}^{\prime}}\left(k_{n t}, K_{t}\right)=\widetilde{W}_{k_{n t} k_{n t}}^{\prime \prime}\left(k_{n t}, K_{t}\right) \dot{k}_{n t}+\widetilde{W}_{k_{n t}}^{\prime}\left(k_{n t}, K_{t}\right) r+\widetilde{W}_{K_{t} k_{n t}}^{\prime \prime}\left(k_{n t}, K_{t}\right) \dot{K}_{t}
$$

Substituting in this expression $\dot{k}_{n t}$ and $\dot{K}_{t}$ by $\tilde{g} k_{n t}$ and $\tilde{g} K_{t}$, taking into account (66) and the last equation in (67), the modified Ramsey rule (34) follows.

Logarithmic utility: $\sigma=1$ 
The value function along a balanced growth path can be expressed as

$$
\begin{aligned}
\widetilde{W}^{1}\left(k_{n t}, K_{t}\right)= & \int_{t}^{\infty} \ln \left[c_{n t} P_{t}^{-\phi} e^{\tilde{g}^{1}[1+\phi(\gamma-\lambda)](\tau-t)}\right] \theta(\tau-t) d \tau= \\
& \ln \left[c_{n t} P_{t}^{-\phi}\right] \int_{t}^{\infty} \theta(\tau-t) d \tau+\tilde{g}^{1}[1+\phi(\gamma-\lambda)] \int_{t}^{\infty}(\tau-t) \theta(\tau-t) d \tau= \\
& \frac{1}{\hat{\rho}} \ln \left[\psi_{\mathrm{C}} \psi_{\mathrm{A}}^{\phi \gamma}\right]+\frac{1}{\hat{\rho}} \ln k_{n t}+\frac{\phi(\gamma-\lambda)}{\hat{\rho}} \ln K_{t}+\frac{\tilde{g}^{1} \bar{J}_{0}[1+\phi(\gamma-\lambda)]}{\hat{\rho}} .
\end{aligned}
$$

Therefore, the value function is defined as

$$
\widetilde{W}^{1}\left(k_{n t}, K_{t}\right)=\widetilde{W}_{0}^{1}+\tilde{\eta}_{1} \ln k_{n t}+\tilde{\eta}_{2} \ln K_{t}=\widetilde{W}_{0}^{1}+\ln \left[k_{n t}^{\tilde{\eta}_{1}} K_{t}^{\tilde{n}_{2}}\right],
$$

with

$$
\widetilde{W}_{0}^{1}=\frac{\tilde{g}^{1}[1+\phi(\gamma-\lambda)] \bar{J}_{0}+\ln \left[\psi_{\mathrm{C}} \psi_{\mathrm{A}}^{\phi \gamma}\right]}{\hat{\rho}}, \quad \tilde{\eta}_{1}=\frac{1}{\hat{\rho}}, \quad \tilde{\eta}_{2}=\frac{\phi(\gamma-\lambda)}{\hat{\rho}} .
$$

On the other hand, the LHS of the Bellman equation can be written as

$$
\int_{t}^{\infty}\left[\ln c_{n t}(\tau) P_{t}^{-\phi}(\tau)\right] \theta(\tau-t) \rho(\tau-t) d \tau=\hat{\rho} \widetilde{W}^{1}\left(k_{n t}, K_{t}\right)+\tilde{g}^{1}[1+\phi(\gamma-\lambda)]\left\{\frac{1}{\hat{\rho}}-\bar{J}_{0}\right\} .
$$

Plugging this into (63), the Bellman equation can be rewritten as

$\hat{\rho} \widetilde{W}^{1}\left(k_{n t}, K_{t}\right)+\tilde{g}^{1}[1+\phi(\gamma-\lambda)]\left\{\frac{1}{\hat{\rho}}-\bar{J}_{0}\right\}=\ln c_{n t}-\phi \ln P_{t}+\left(\widetilde{W}_{k_{n t}}^{1}\right)^{\prime}(t)\left[r k_{n t}-c_{n t}\right]+\left(\widetilde{W}_{K_{t}}^{1}\right)^{\prime}(t) \dot{K}_{t}$.

And differentiating with respect $k_{n t}$, one gets

$$
\hat{\rho}\left(\widetilde{W}_{k_{n t}}^{1}\right)^{\prime}\left(k_{n t}, K_{t}\right)=\left(\widetilde{W}_{k_{n t} k_{n t}}^{1}\right)^{\prime \prime}\left(k_{n t}, K_{t}\right) \dot{k}_{n t}+\left(\widetilde{W}_{k_{n t}}^{1}\right)^{\prime}\left(k_{n t}, K_{t}\right) r+\left(\widetilde{W}_{K_{t} k_{n t}}^{1}\right)^{\prime \prime}\left(k_{n t}, K_{t}\right) \dot{K}_{t}
$$

Taking into account (72), equation (73) leads to

$$
\hat{\rho}=-\frac{\dot{k}_{n t}}{k_{n t}}+r,
$$

and substituting in this expression $\dot{k}_{n t}$ by $\tilde{g}^{1} k_{n t}$ expression (35) follows.

\section{Proof of Proposition 9.}

In the central-planner balanced path equilibrium, when $\sigma=1, g^{1 *}=g_{\hat{\rho}}^{1 *}, \psi_{A}^{1 *}=\psi_{A \hat{\rho}}^{1 *}$ and the associated consumer welfare is

$$
\begin{aligned}
W^{1}\left(K_{t}\right)= & \ln \left(c_{t}^{*} P_{t}^{-\phi}\right) \int_{t}^{\infty} \theta(\tau-t) d \tau+g^{1 *}[1+\phi(\gamma-\lambda)] \int_{t}^{\infty}(\tau-t) \theta(u-t) d \tau= \\
& \frac{\ln \left(c_{t}^{*} P_{t}^{-\phi}\right)}{\hat{\rho}}+\frac{g^{1 *}[1+\phi(\gamma-\lambda)] \bar{J}_{0}}{\hat{\rho}}=W_{\hat{\rho}}^{1}\left(K_{t}\right)+\frac{g^{1 *}[1+\phi(\gamma-\lambda)]}{\hat{\rho}}\left(\bar{J}_{0}-\frac{1}{\hat{\rho}}\right) .
\end{aligned}
$$


Likewise, in the market economy, $\tilde{g}^{1 *}=\tilde{g}_{\hat{\rho}}^{1 *}, \tilde{\psi}_{\mathrm{A}}=\tilde{\psi}_{\mathrm{A} \hat{\rho}}=(\tilde{\alpha} \gamma B)^{\frac{1}{1-\alpha \gamma}}$, and then, following the same reasoning

$$
\widetilde{W}^{1}\left(K_{t}\right)=\widetilde{W}_{\hat{\rho}}^{1}\left(K_{t}\right)+\frac{\tilde{g}^{1 *}[1+\phi(\gamma-\lambda)]}{\hat{\rho}}\left(\bar{J}_{0}-\frac{1}{\hat{\rho}}\right) .
$$

From Lemma 15 it is known that $\Omega(g, \eta)<\bar{J}$, and particularizing for $\sigma=1, \Omega(g, 0)<\bar{J}_{0}$. Given that $\Omega(g, 0)=1 / \hat{\rho}$, the last term in the expression above is positive.

\section{Proof of Proposition 12.}

The modified Ramsey rule under a central-planner or a market economy can be written as

$$
g\left(\psi_{\mathrm{A}}\right)=r\left(\psi_{\mathrm{A}}\right)-\frac{1}{\Omega(g, \eta)}, \quad \tilde{g}\left(\psi_{\mathrm{A}}\right)=\tilde{r}\left(\psi_{\mathrm{A}}\right)-\frac{1}{\Omega(g, \eta)} .
$$

By linearizing $g\left(\psi_{\mathrm{A}}\right)$ at $\psi_{\mathrm{A}}=\tilde{\psi}_{\mathrm{A}}^{N T *} \equiv(\tilde{\alpha} \gamma B)^{\frac{1}{1-\alpha \gamma}}$, where superscript NT denotes the market economy with no taxes or subsidy, one gets $^{16}$

$$
g\left(\psi_{\mathrm{A}}\right) \simeq g\left(\tilde{\psi}_{\mathrm{A}}^{N T *}\right)+\frac{r^{\prime}\left(\tilde{\psi}_{\mathrm{A}}^{N T *}\right)}{1+\eta_{\bar{\Omega}(g, \eta)}}\left(\psi_{\mathrm{A}}-\tilde{\psi}_{\mathrm{A}}^{N T *}\right) .
$$

Moreover, taking into account $(20)$ and (30) it follows that $g\left(\tilde{\psi}_{\mathrm{A}}^{N T *}\right)=\tilde{g}\left(\tilde{\psi}_{\mathrm{A}}^{N T *}\right)$. In consequence, evaluating (74) at $\psi_{\mathrm{A}}^{*}$, the gap in the growth rates under the centralized and the market economy without taxes can be approximated as

$$
g^{*}-\tilde{g}^{N T *}=g\left(\psi_{\mathrm{A}}^{*}\right)-\tilde{g}\left(\tilde{\psi}_{\mathrm{A}}^{N T *}\right) \simeq \frac{r^{\prime}\left(\tilde{\psi}_{\mathrm{A}}^{N T *}\right)}{1+\eta_{\overline{\Omega(g, \eta)}} \overline{\bar{J}}}\left(\psi_{\mathrm{A}}^{*}-\tilde{\psi}_{\mathrm{A}}^{N T *}\right) .
$$

Applying identical reasoning for the case of constant discounting, the gap between the growth rates reads

$$
g_{\hat{\rho}}^{*}-\tilde{g}_{\hat{\rho}}^{N T *}=g_{\hat{\rho}}\left(\psi_{\mathrm{A} \hat{\rho}}^{*}\right)-\tilde{g}_{\hat{\rho}}\left(\tilde{\psi}_{\mathrm{A}}^{N T *}\right) \simeq \frac{r^{\prime}\left(\tilde{\psi}_{\mathrm{A}}^{N T *}\right)}{1+\eta}\left(\psi_{\mathrm{A} \hat{\rho}}^{*}-\tilde{\psi}_{\mathrm{A}}^{N T *}\right) .
$$

Recall that under constant or non-constant discounting $\tilde{\psi}_{\mathrm{A}}^{N T *}=(\tilde{\alpha} \gamma B / \lambda)^{1 /(1-\alpha \gamma)}$.

The total effect of taxes on the market growth rate is

$$
\begin{gathered}
\frac{d \tilde{g}}{d T}=\frac{\partial \tilde{g}}{\partial \psi_{\mathrm{A}}} \frac{d \psi_{\mathrm{A}}}{d T}+\frac{\partial \tilde{g}}{\partial T}=\frac{\frac{\partial \tilde{r}}{\partial \psi_{\mathrm{A}}} \frac{d \psi_{\mathrm{A}}}{d T}+\frac{\partial \tilde{r}}{\partial T}}{1+\eta \frac{\bar{J}}{\Omega(g, \eta)}}=\frac{\Psi}{1+\eta \frac{\bar{J}}{\Omega(g, \eta)}}, \\
\frac{d \tilde{g}_{\hat{\rho}}}{d T}=\frac{\partial \tilde{g}_{\hat{\rho}}}{\partial \psi_{\mathrm{A}}} \frac{d \psi_{\mathrm{A}}}{d T}+\frac{\partial \tilde{g}_{\hat{\rho}}}{\partial T}=\frac{\frac{\partial \tilde{r}}{\partial \psi_{\mathrm{A}}} \frac{d \psi_{\mathrm{A}}}{d T}+\frac{\partial \tilde{r}}{\partial T}}{1+\eta}=\frac{\Psi}{1+\eta} .
\end{gathered}
$$

\footnotetext{
${ }^{16}$ Note that the Ramsey curve is inverse U-shaped and $(\alpha \gamma B)^{1 /(1-\alpha \gamma)}$ is lower than $\hat{\psi}_{\mathrm{A}}=$ $\left(\alpha \beta \gamma^{2} B / \lambda\right)^{1 /(1-\alpha \gamma)}$ (where the Ramsey curve reaches its maximum). Hence, the linear approximation is inaccurate for values of $\psi_{\mathrm{A}}>\hat{\psi}_{\mathrm{A}}$.
} 
Lemma 15 states that $\bar{J}>\Omega(g, \eta)$, then for $\sigma<1$ it follows that

$$
\left|\frac{d\left(g^{*}-\tilde{g}^{N T *}\right)}{d T} \frac{T}{g^{*}-\tilde{g}^{N T *}}\right|<\left|\frac{d\left(g_{\hat{\rho}}^{*}-\tilde{g}_{\hat{\rho}}^{N T *}\right)}{d T} \frac{T}{g_{\hat{\rho}}^{*}-\tilde{g}_{\hat{\rho}}^{N T *}}\right|
$$

which is equivalent to

$$
\frac{\Psi}{\psi_{\mathrm{A}}^{*}-\tilde{\psi}_{\mathrm{A}}^{N T *}}<\frac{\Psi}{\psi_{\mathrm{A} \hat{\rho}}^{*}-\tilde{\psi}_{\mathrm{A}}^{N T *}} \Leftrightarrow \psi_{\mathrm{A} \hat{\rho}}^{*}<\psi_{\mathrm{A}}^{*},
$$

which is true from Proposition 6. 


\section{Legends}

Figure 1: Constant/non-constant discount functions \& instantaneous discount rates.

Figure 2: Equilibria under constant \& non-constant discounting.

Figure 3: $\alpha-\tilde{\alpha}$ large w.r.t. $\phi$.

Figure 4: $\phi$ large w.r.t. $\alpha-\tilde{\alpha}$.

Figure 5: $\widetilde{W}^{1}\left(K_{t}\right)>W^{1}\left(K_{t}\right)$ for different $\tilde{\alpha}$.

Figure 6: $\widetilde{W}^{1}\left(K_{t}\right)>W^{1}\left(K_{t}\right)$ for different $\phi$.

Figure 7: $\widetilde{W}^{1}\left(K_{t}\right)>W^{1}\left(K_{t}\right)$ for different $\lambda$.

Figure 8: $\widetilde{W}^{1}\left(K_{t}\right)>W^{1}\left(K_{t}\right)$ for different $\gamma$.

\begin{tabular}{|c|c|c|c|c|}
\hline & \multirow{2}{*}{$\alpha$} & \multirow{2}{*}{$g^{1 *}-\tilde{g}^{1 *}$} & \multirow{2}{*}{$W_{\hat{\rho}}^{1}-\widetilde{W}_{\hat{\rho}}^{1}$} & $W^{1}-\widetilde{W}^{1}$ \\
\hline & & & & $\rho=1.2 \times 10^{-3}, \varphi=0.9, \delta=1.39$ \\
\hline Benchmark & 0.5 & -0.0069 & 44.5 & -14 \\
\hline$\widetilde{\alpha} \rightarrow 0.41$ & 0.5 & -0.0076 & 42.1 & -22.9 \\
\hline$\phi \rightarrow 3$ & 0.5 & -0.0151 & 72.9 & -74 \\
\hline$\lambda \rightarrow 0.78$ & 0.45 & -0.0062 & 32.9 & -21.1 \\
\hline$\gamma \rightarrow 1.01$ & 0.48 & -0.007 & 39 & -21.5 \\
\hline
\end{tabular}

Table 1: Comparing centralized and market economies 